\title{
Disentangling how multiple traits drive 2 strain frequencies in SIS dynamics with coinfection
}

\author{
Thi Minh Thao Le ${ }^{1, *}$, Sten Madec ${ }^{1,+}$, Erida Gjini ${ }^{2, \dagger}$ \\ ${ }^{1}$ Laboratory of Mathematics, University of Tours, Tours, France, \\ ${ }^{2}$ Center for Stochastic and Computational Mathematics, Instituto Superior Tecnico, Lisbon, Portugal \\ *Thi-minh-thao.Le@lmpt.univ-tours.fr, ${ }^{+}$Sten.Madec@lmpt.univ-tours.fr, ${ }^{\dagger}$ erida.gjini@tecnico.ulisboa.pt
}

\begin{abstract}
A general theory for competitive dynamics among many strains at the epidemiological level is required to understand polymorphisms in virulence, transmissibility, antibiotic resistance and other biological traits of infectious agents. Mathematical coinfection models have addressed specific systems, focusing on the criteria leading to stable coexistence or competitive exclusion, however, due to their complexity and nonlinearity, analytical solutions in coinfection models remain rare. Here we study a 2-strain SIS compartmental model with co-infection/co-colonization, incorporating multiple fitness dimensions under the same framework: variation in transmissibility, duration of carriage, pairwise susceptibilities to coinfection, coinfection duration, and transmission priority effects from mixed coinfection. Taking advantage of a singular perturbation approach, under the assumption of strain similarity, we expose how strain dynamics on a slow timescale are explicitly governed by a replicator equation which encapsulates all traits and their interplay. This allows us to predict explicitly not only the final epidemiological outcome of a given 2-player competition, but moreover, their entire frequency dynamics as a direct function of their relative variation and of strain-transcending global parameters. Based on mutual invasion fitnesses, we analyze and report rigorous results on transition phenomena in the 2-strain system, strongly mediated via coinfection prevalence. This framework offers a deeper analytical understanding of 2-strain competitive games in coinfection, with applications to virulence, interventions, antibiotic resistance, and social evolution theory.
\end{abstract}

Keywords: SIS model; coinfection; polymorphism; replicator equation; timescale separation; strain similarity; invasion fitness; coexistence; competitive release; epidemiology; priority effects; fitness cost

\section{Introduction}

Epidemiological models of coinfection have a long history of study (Levin and Pimentel, 1981; Adler and Brunet, 1991; Nowak and May, 1994, May and Nowak, 1995, van Baalen and Sabelis, 1995, Mosquera and Adler, 1998; Martcheva, 2009: Thieme, 2007, Alizon, 2013). Examples of multi-strain infectious agents where coinfection processes appear and shape epidemiology include Streptococcus pneumoniae bacteria (Lipsitch 1997; Gjini et al., 2016), Bordetella pertussis (Nicoli et al., 2015), Mycobacterium tuberculosis (Cohen et al. 2012), Staphylococcus Aureus, (Pinotti et al. 2019) and many others, comprising plants (Susi et al., 2015 Halliday et al. 2020), and also inter-species co-colonization such as between Haemophilus Influenzae and pneumococcus serotypes (Margolis et al., 2010, Cobey and Lipsitch, 2013) and coinfection with different viruses (Furuya-Kanamori et al., 2016). Typically the strain-defining parameters vary much less within than between species. However, until now, models have not leveraged the conceptual and analytic advantages of strain similarity to the full extent, except for the classical comparisons between strain-specific basic reproduction numbers.

While it has long been recognized that in coinfection systems, basic reproduction numbers alone do not determine strain competitive dynamics (Nowak and May, 1994, van Baalen and Sabelis, 1995), a generic framework to integrate variation among strains along several phenotypic axes and coinfection, and map these directly to strain frequencies, has not been developed. Moreover, until now simplified versions of traits involved in SIS dynamics between two strains have been addressed: either modeling vulnerability to coinfection as a single parameter (Alizon, 2013), or focusing just on cross-strain competition (Lipsitch, 1997), four-way competitive interactions via altered susceptibilities to coinfection (Gjini et al., 2016), exclusively cooperative dynamics (Chen et al. 2017), or focusing on transmission and clearance rate variation (Martcheva, 2009 Thieme, 2007).

A key level of strain interactions in coinfection is the within-host level, where the order and timing of arrival, can matter for onward transmission or clearance. Such interactions when studied empirically have revealed strong priority effects, where the first arriving genotype has an advantage over later arriving ones 
(De Roode et al., 2005, Halliday et al., 2020). Independently of the underlying mechanisms, whether via host immunity, resource overlap within host or others, priority effects have repercussions on disease dynamics and parasite assemblage dynamics at higher scales. Yet, the full extent of the inter-dependence between this trait and other traits involved in epidemiological dynamics remains poorly understood. Thus, although several aspects of coinfection have been studied, typically with simulations or analyses restricted to special cases and particular models, a comprehensive and concise theoretical framework for how coinfection prevalence broadly interplays with multiple traits between 2 coinfecting agents in endemic systems is still missing.

In this paper, we describe and study a general system for epidemiological dynamics of similar co-infecting entities (e.g. strains of the same infectious agent or similar species) that comprise a rich ecological and epidemiological phenomenology. Such a system could apply, but not be limited to, polymorphic Streptococcus pneumonia bacteria or other commensal bacteria (Lipsitch, 1997, Gjini et al., 2016, Davies et al., 2019). We generalize a previously- introduced quasi-neutral Susceptible-Infected-Susceptible (SIS) framework for 2 circulating strains, where we showed that asymmetries in pairwise susceptibilities to co-colonization create frequency-dependent advantage for one of the strains and can give rise to coexistence, bistability as well as competitive exclusion (Gjini et al., 2016, Gjini and Madec, 2017). Here, we study additional variation between two microbial strains, in other traits besides vulnerability to co-colonization, including transmission rate and duration of carriage, two classical traits that are known to vary among pneumococcus serotypes (Abdullahi et al. 2012), but in general can also vary among two arbitrary infectious agents, e.g. an antibiotic-resistant and an antibiotic-sensitive strain. Furthermore, we also allow for variation in transmission biases from coinfected hosts carrying a mixture of two strains, and in duration of coinfection episodes, where priority effects can play a role. Until now analytic solutions for how such systems behave in time have not been obtained, although theoretical models have studied the conditions for coexistence vs. competitive exclusion or used numerical simulations as an approach in specific cases (Lipsitch, 1997; Zhang et al., 2004, Gjini et al., 2016 Alizon, 2008; Hansen and Day, 2014).

The novelty of our approach lies in applying singular perturbation theory to a quasi-neutral model, whereby we obtain a timescale separation, similar to Gjini and Madec (2017), in order to express the total dynamics as a fast plus a slow component, related to broken symmetries along 5 traits between strains: transmission rate $\beta_{i}$, clearance rate $\gamma_{i}$, coinfection duration $\gamma_{i j}$, co-colonization interactions $k_{i j}$, and transmission priority effects from coinfection $p_{i j}^{i}$. Net competition dynamics can be complex because all traits interact nonlinearly to determine final strain fitness at the host population level, but here we make such selective process entirely explicit. Moreover, we show how the strain-transcending parameters, defining the neutral model at the center, feed back on the strain dynamics on the slow timescale, and tune the net importance of each phenotypic axis.

We concentrate only on up to 2 strains co-colonizing a host $(\mathrm{MOI}=2)$, assuming that primary colonization by one strain alters host susceptibility to secondary strains, (increasing or decreasing it) by a factor $k_{i j}$, relative to uninfected hosts, without acquired immunity. We apply results for the $N$-strain case derived in (Le et al., 2021). The altered susceptibilities to co-colonization, given by a $2 \times 2$ matrix in the case of 2 strains, can comprise antagonistic or facilitative interactions $\left(k_{i j}<1\right.$ or $\left.\geq 1\right)$. Beyond enabling competition and cooperation to be studied under the same framework, our model allows also for any asymmetries in this co-colonization susceptibility matrix, as for the coinfection clearance rate matrix, depending on strain composition, and for transmission biases from coinfected hosts, depending on order of strain arrival.

Considering the complex epidemiological multi-strain dynamics in fast and slow components has many analytic advantages as argued in (Madec and Gjini, 2020, Le et al., 2021). Our neutral model satisfies the criteria for ecological and population-genetic neutrality discussed in the context of 'no coexistence for free' (Lipsitch et al. 2009), but much more than a neutral null model, our approach highlights the neutral model as the core organizing centre of the multi-strain dynamics. This is made entirely explicit via the slow-fast timescale separation formalism (Madec and Gjini, 2020, Le et al., 2021), linking neutral and non-neutral dynamics in an 'organic' manner, and showing how parameters of the neutral model impact on the slow 'selection' dynamics between strains, driven by specific feedbacks.

The paper is organized as follows. First, we describe the epidemiological framework. Secondly, we expose and elaborate on a closed and generic analytic solution for 2-strain frequency dynamics over the slow time-scale, in a changing fitness landscape shaped by multi-trait variation. This solution coincides with a version of the classical replicator equation in 2 dimensions (Hofbauer and Sigmund, 2003), but with an explicit payoff matrix derived from 5-dimensional trait variation between strains relatively weighted in the overall pairwise invasion fitness (Le et al. 2021). Third, we analyze why and how coexistence, bistability or competitive exclusion of either strain may occur between any two strains, for a fixed given trait variation between them. Fourth, we focus our attention on an in-depth analytic investigation of how strain-transcending mean-field gradients can shift the same system across these regimes, for different values of global $R_{0}$ or coinfection prevalence, detailing the context-dependence of net outcomes. Finally, we conclude with a roadmap for biological applications. We believe our analysis and approach offer a fresh perspective, to quantify and predict how multiple traits together shape strain dynamics and final equilibrium prevalence via joint population feedback. 
a

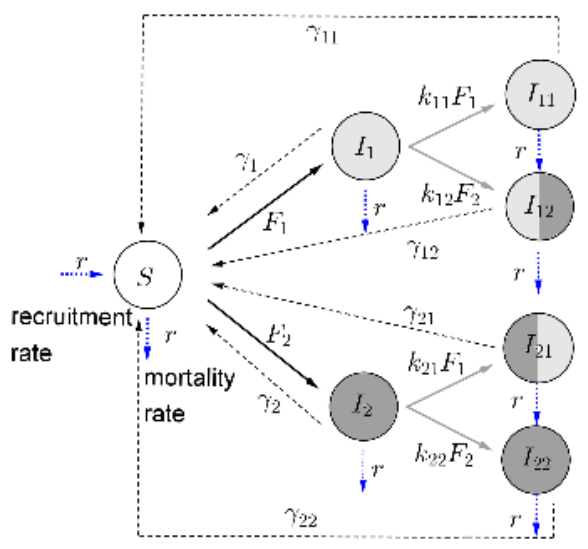

C

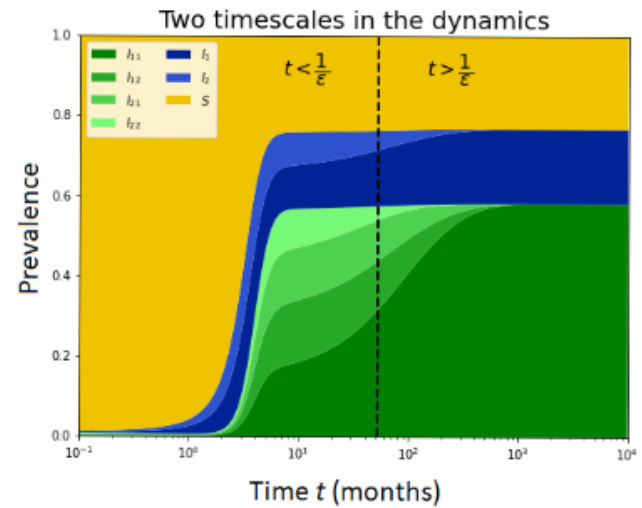

b

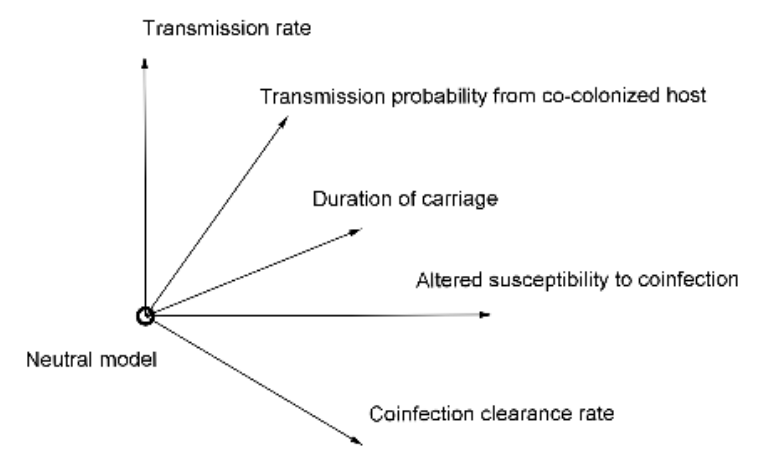

d

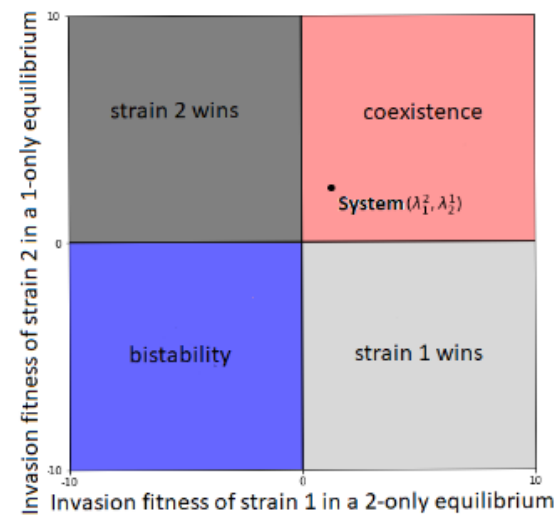

Figure 1: SIS co-colonization model diagram for two strains $(N=2)$. a. The model follows the structure in (Gjini and Madec, 2017) but here two strains can differ in transmission rate $\beta_{i}$, clearance rate $\gamma_{i}$, co-colonization clearance rate $\gamma_{i j}$, altered susceptibilities to co-colonization $k_{i j}$ and transmission biases from coinfection $p_{i j}^{i}$. Thus any combination of relative fitness costs and advantages can be encapsulated, provided that their variation is not too big, as expected for similar conspecific strains, or similar infectious co-circulating 'species'. Non-carriers $(S)$ become carriers of either strain 1 or $2\left(I_{i}\right)$ with force of infection $F_{i}=\beta_{i}\left(I_{i}+I_{i i}+\sum_{i j}\left(p_{i j}^{i} I_{i j}+p_{j i}^{i} I_{j i}\right)\right)$ where the mixed carriage compartment $\left(I_{i j}\right)$ may transmit either strain with a slightly biased probability away from $1 / 2$ depending on the order of arrival (see (Le et al., 2021)). Here $1 / \gamma_{i}$ is the strain-specific duration of single colonization, $1 / \gamma_{i j}$ are the composition-specific durations of co-colonization, which can vary for all four $I_{i j}$ classes. The coefficients $k_{i j}$ capture the altered relative susceptibilities to co-colonization between strains, when a host is already colonized, and transitions from primary colonization to co-colonization. The parameter $r$ is the natural birth/death rate of the host. b. Assuming strain similarity, the epidemiological dynamics in such an SIS co-colonization model, can be decomposed into a fast (neutral) component and slow (non-neutral) component. The slow dynamics follow an explicit replicator equation which includes in the net payoff matrix variation across 5 dimensions of fitness for each strain. This equation allows to predict analytically the entire temporal dynamics of two strains as a function of their epidemiological phenotypes. c. We simulate an example of 2 -strain system in two timescales. On the fast time-scale $(o(1 / \epsilon))$, strains follow neutral dynamics, driven by mean-field parameters, where total prevalence of susceptibles, single infection and co-infection stabilize. On a slow time-scale, $\epsilon$, within conserved global epidemiological compartments, complex nonneutral dynamics between strains takes place, depicted here by the blue and green shadings. d. Each system can be in one of four scenarios between 2 strains, depending on the signs of mutual invasion fitnesses (e.g. dynamics in c corresponds to the black point in the coexistence region). We find that frequency dynamics are explicitly governed by the $\lambda_{i}^{j}$. In our model, invasion fitnesses are explicit functions of strain variability and global mean-field parameters.

\section{The modeling framework}

\subsection{The SIS model with coinfection}

We study an infectious agent transmitted in a host population following Susceptible-Infected-Susceptible dynamics, where there are two co-circulating strains (denoted by 1 and 2). Susceptible hosts $S$ can acquire any strain $i$, by which they enter the single colonization compartment $I_{i}$. Already colonized hosts $I_{i}$ can acquire any secondary strain $j$, leading them to enter the co-colonization compartment $I_{i j}$. As in the pioneering model by (van Baalen and Sabelis, 1995), an important epidemiological feature here is that hosts can be coinfected twice by the same strain $\left(I_{11}\right.$ and $I_{22}$ compartments). Without this assumption, a rare strain always has an advantage: it can infect hosts already infected by the common strain while the common strain has few hosts 
to coinfect. Co-colonized and singly-colonized hosts transmit at equal total rate, and hosts carrying a mixture of two different strains transmit any strain $i$ with a given probability $p_{i j}^{i}$ which can be different from $1 / 2$ and may depend on the order of arrival within-host (e.g. $p_{i j}^{i} \neq p_{j i}^{i}$ ). The model follows the structure in (Gjini et al. 2016, Gjini and Madec, 2017), but here we have a more general model, allowing for more trait variation between strains. In (Gjini and Madec, 2017), only pairwise interactions in co-colonization were modeled as different between strains ( $k_{i j}$ coefficients), and this was sufficient to generate stabilizing mechanisms for coexistence. Thus, in addition to $k_{i j}$, we model strain-specific transmission rate, $\beta_{i}$, and clearance rate $\gamma_{i}$, as well as coinfection clearance rates $\gamma_{i j}$ and transmission biases from coinfection $p_{i j}^{i}$. Recruitment of susceptibles happens at per-capita rate $r$, assumed equal to the natural mortality rate. The scheme of the model for two strains is given in Figure 1. The explicit dynamical system of equations for the $N$-strain version of this epidemiological model is derived in Le et al. (2021), and given by:

$$
\left\{\begin{array}{l}
\frac{d S}{d t} \quad=r(1-S)+\sum_{i=1}^{N} \gamma_{i} I_{i}+\sum_{i, j=1}^{N} \gamma_{i j} I_{i j}-S \sum_{i=1}^{N} F_{i}, \\
\frac{d I_{i}}{d t} \quad=F_{i} S-\left(r+\gamma_{i}\right) I_{i}-I_{i} \sum_{j=1}^{N} k_{i j} F_{j}, \quad 1 \leq i \leq N, \\
\frac{d I_{i j}}{d t} \quad=k_{i j} I_{i} F_{j}-\left(r+\gamma_{i j}\right) I_{i j}, \quad 1 \leq i, j \leq N,
\end{array}\right.
$$

where in our case for $N=2, i, j \in\{1,2\}$, and the force of infection for each strain is given by:

$$
F_{i}=\beta_{i}\left(I_{i}+\sum_{j=1}^{N}\left(p_{i j}^{i} I_{i j}+p_{j i}^{i} I_{j i}\right)\right),
$$

Some conventions and notations for the parameters in a multiple-trait model, under the similarity assumption between strains, are given in Table 1. To obtain the fast-slow decomposition, we rewrite the system in terms of new aggregate variables as in (Madec and Gjini, 2020), such as the total prevalence of colonized hosts $T$, the total prevalence of hosts transmitting either strain $J_{i}$, the total prevalence of single colonization $I$, and co-colonization $D$ :

$$
T=\sum_{i=1}^{2} I_{i}+\sum_{i, j=1}^{2} I_{i j}, \quad J_{i}=I_{i}+\sum_{j=1}^{2}\left(p_{i j}^{i} I_{i j}+p_{j i}^{i} I_{j i}\right), \quad I=\sum_{i=1}^{N} I_{i}, \quad D=T-I .
$$

Following similar technical steps as in (Madec and Gjini 2020), in Le et al. (2021), we have derived that during the fast timescale, strains behave as neutral (all parameters are identical between them) and each global aggregated variable tends to its equilibrium: $S \rightarrow S^{*}, T \rightarrow T^{*}, I \rightarrow I^{*}$ and $D \rightarrow D^{*}$ as $\epsilon \rightarrow 0$. With the basic reproduction number in this system denoted $R_{0}=\frac{\beta}{m}$, this equilibrium is given by:

$$
S^{*}=\frac{1}{R_{0}}, \quad T^{*}=1-S^{*}, \quad I^{*}=\frac{T^{*}}{1+k\left(R_{0}-1\right)}, \quad D^{*}=T^{*}-I^{*}
$$

and the ratio of single to co-colonization is given by $\mu=\frac{I^{*}}{D^{*}}=\frac{1}{k\left(R_{0}-1\right)}$.

Further, during the slow time scale $\epsilon t$, strains are not equivalent, their differences in fitness start to get manifested, and what follows is non-neutral dynamics at the level of strain frequencies $z_{i}$. For a 2-strain system, there are four possibilities for the equilibrium: i) coexistence, ii-iii) exclusion of each strain, and iv) bistability of competitive exclusion states, also known as a priority effect. Below, these outcomes and their dynamics are shown to depend explicitly on mutual invasion growth rates between two strains.

\subsection{Pairwise invasion fitness and replicator dynamics via timescale separation}

In this more complex model, we follow the same reasoning as in (Madec and Gjini, 2020), focusing on mutual invasion fitnesses, to express the selective dynamics occurring on the slow time scale. We will define $\lambda_{i}^{j}$ to be invasion fitness of strain $i$ in an equilibrium set by strain $j$ alone, a classical approach in adaptive dynamics (Geritz et al., 1998). Initially, based on Le et al. (2021), we redefine $\theta_{i}$ via the global quantities and parameters of the neutral model:

$$
\theta_{1}=\left(\frac{T^{*}}{D^{*}}\right)^{2}, \quad \theta_{2}=\frac{\gamma I^{*}\left(I^{*}+T^{*}\right)}{2 m T^{* 2}}\left(\frac{T^{*}}{D^{*}}\right)_{4}^{2}, \quad \theta_{3}=\frac{\gamma T^{*}}{2 m D^{*}}, \quad \theta_{4}=\frac{T^{*}}{D^{*}}, \quad \theta_{5}=\frac{\beta I^{*} T^{*}}{2 m D^{*}} .
$$


Table 1: Conventions and notations of parameters and variables, where we assume the traits are numerically close for closely-related strains, or similar infectious entities. This similarity assumption $(0<\epsilon \ll 1$, small) forms the basis for dynamic decomposition into fast and slow components $(\mathrm{Le}$ et al. 2021).

\begin{tabular}{|c|c|c|c|}
\hline Parameter & Interpretation & Specification & Features \\
\hline \multicolumn{4}{|l|}{$\begin{array}{l}\text { Original system } \\
\text { (Quasi-neutral) }\end{array}$} \\
\hline$\gamma_{i}=\gamma\left(1+\epsilon \nu_{i}\right)$ & $\begin{array}{l}\text { Strain-specific clearance rates of sin- } \\
\text { gle colonization }\end{array}$ & $\Delta \nu=\nu_{2}-\nu_{1}$ & Favours 1 if $\Delta \nu>0$ \\
\hline$\gamma_{i j}=\gamma\left(1+\epsilon u_{i j}\right)$ & $\begin{array}{l}\text { Clearance rates of co-colonization } \\
\text { with } i \text { and } j\end{array}$ & $\Delta_{i} u=2 u_{j j}-\left(u_{i j}+u_{j i}\right)$ & Favours $i$ if $\Delta_{i} u>0$ \\
\hline$p_{i j}^{s}=\frac{1}{2}+\epsilon \omega_{i j}^{s}$ & $\begin{array}{l}\text { Transmission probability }{ }^{1} \text { of } s \in \\
\{i, j\} ; \text { from a host co-colonized by } \\
\text { strain- } i \text { then }-j \text { (priority effects). }\end{array}$ & $\Delta \omega=\omega_{12}^{1}-\omega_{21}^{2}$ & Favours 1 if $\Delta \omega>0$ \\
\hline$k_{i j}=k+\epsilon \alpha_{i j}$ & $\begin{array}{l}\text { Relative factor of altered suscepti- } \\
\text { bility to co-colonization between col- } \\
\text { onizing strain } i \text { and co-colonizing } \\
\text { strain } j\end{array}$ & $\Delta_{i} \alpha=\alpha_{j i}-\alpha_{j j}+\mu\left(\alpha_{j i}-\alpha_{i j}\right)$ & Favours $\mathrm{i}$ if $\Delta_{i} \alpha>0$ \\
\hline \multicolumn{4}{|l|}{$\begin{array}{l}\text { Embedded } \\
\text { neutral system } \\
\text { (Fast): }\end{array}$} \\
\hline$r$ & Susceptible recruitment rate & Equal to natural mortality & Host turnover $\uparrow$ \\
\hline$\beta$ & Transmission rate (infectiousness) & $\beta>0$ & Transmission $\uparrow$ \\
\hline$\gamma$ & Clearance rate of single colonization & $\begin{array}{l}\text { Equal to clearance rate of co- } \\
\text { colonization, } \gamma>0\end{array}$ & Transmission $\downarrow$ \\
\hline$m$ & Net infected host turnover rate & $m=r+\gamma$ & $R_{0} \downarrow$ \\
\hline$R_{0}$ & Basic reproduction number & $R_{0}=\beta / m>1$ & Colonization $\uparrow$ \\
\hline$k$ & $\begin{array}{l}\text { Altered susceptibility to co- } \\
\text { colonization when colonized }\end{array}$ & & $\begin{array}{l}k \geq 1: \text { facilitation } \\
k<1: \text { competition }\end{array}$ \\
\hline$\mu$ & Single to co-colonization ratio & $\mu=\frac{1}{\left(R_{0}-1\right) k}$ & Monotone in $R_{0}$ and $k$ \\
\hline \multicolumn{4}{|l|}{$\begin{array}{l}\text { Non-neutral } \\
\text { system (Slow): }\end{array}$} \\
\hline$\theta_{1}$ & Weight of transmission rate axis $\beta_{i}$ & $(\mu+1)^{2}$ & Depends on $\mu$ \\
\hline$\theta_{2}$ & Weight of clearance rate axis $\gamma_{i}$ & $\frac{\gamma}{2(\gamma+r)}\left(2 \mu^{2}+\mu\right)$ & Depends on $\mu, \gamma, r$ \\
\hline$\theta_{3}$ & $\begin{array}{l}\text { Weight of coinfection clearance rate } \\
\text { axis } \gamma_{i j}\end{array}$ & $\frac{\gamma}{2(\gamma+r)}(\mu+1)$ & Depends on $\mu, \gamma, r$ \\
\hline$\theta_{4}$ & $\begin{array}{l}\text { Weight of transmission priority ef- } \\
\text { fects from coinfection axis } p_{i j}^{i}\end{array}$ & $\mu+1$ & Depends on $\mu$ \\
\hline$\theta_{5}$ & $\begin{array}{l}\text { Weight of susceptibilities to coinfec- } \\
\text { tion axis } k_{i j}\end{array}$ & $\frac{1}{2 k}$ & Depends on $k$ \\
\hline
\end{tabular}

As derived in detail in Le et al. (2021), we have that, in our model with several variable traits between strains, for $i, j \in\{1,2\}$, the mutual invasion fitnesses are given by:

$$
\lambda_{i}^{j}=\theta_{1}\left(b_{i}-b_{j}\right)+\theta_{2}\left(-\nu_{i}+\nu_{j}\right)+\theta_{3}\left(-u_{i j}-u_{j i}+2 u_{j j}\right)+\theta_{4}\left(\omega_{i j}^{i}-\omega_{j i}^{j}\right)+\theta_{5}\left(\mu\left(\alpha_{j i}-\alpha_{i j}\right)+\alpha_{j i}-\alpha_{j j}\right) .
$$

This analytic expression sums the relative contributions of multiple trait variations at the same time, with the weighting constants $\theta_{i}$ defined above and given explicitly in Table 1. By the notations of $\lambda_{i}^{j}$, setting 
$\Lambda=\left(\lambda_{i}^{j}\right)_{i, j \in\{1,2\}}$, system 2.1 on the slow timescale $\epsilon t$, can be approximated by the replicator equation

$$
\left\{\begin{array}{l}
\frac{d z}{d \tau}=\Theta z\left(\Lambda z-z^{T} \Lambda z\right) \\
z_{1}+z_{2}+\cdots+z_{N}=1
\end{array}\right.
$$

for variables $z=\left(z_{1}, z_{2}\right)$ denoting strain frequencies, where the overall speed of dynamics $\Theta$ is given by:

$$
\Theta=\frac{2 m D^{* 2}}{2 T^{* 2}-I^{*} D^{*}}=\frac{2 m}{2 \mu^{2}+3 \mu+2} .
$$

When there is just variation in co-colonization coefficients $k_{i j}$, we recover the model and the $\Theta$ in (Madec and Gjini, 2020). Further, recall in (Madec and Gjini, 2020) the term $z^{T} \Lambda z$ is denoted as $Q$ and referred to as mean invasibility of the system, also revisited in terms of colonization resistance (Gjini and Madec, 2021b). In the 2-strain system, more explicitly we have: $Q=z^{T} \Lambda z=\left(\lambda_{1}^{2}+\lambda_{2}^{1}\right) z_{1} z_{2}$, and this quantity is positive only in the case of coexistence between two strains.

In this slow-fast derivation, epidemiological variables of the original model (system 2.1) are then a function of strain frequencies of the slow system:

$$
I_{i}(\tau)=I^{*} z_{i}(\tau) \quad \text { and } \quad I_{i j}(\tau)=D^{*} z_{i}(\tau) z_{j}(\tau)
$$

where $I^{*}$ and $D^{*}$ give the overall prevalence of single and co-colonization in the endemic system (neutral model), and $z_{i}$ and $z_{i} z_{j}$ give the proportions occupied by strain $i$ and the pair of strains $i$ and $j$, in single colonization and co-colonization respectively.

\section{General outcomes of the 2-strain system}

\subsection{Equilibria of the system}

Denote by $z^{*}=\left(z_{1}^{*}, z_{2}^{*}\right)$ the nonzero equilibrium state of (2.7), where strain frequencies are given by:

$$
\left(z_{1}^{*}, z_{2}^{*}\right)=\left(\frac{\lambda_{1}^{2}}{\lambda_{1}^{2}+\lambda_{2}^{1}}, \frac{\lambda_{2}^{1}}{\lambda_{1}^{2}+\lambda_{2}^{1}}\right) .
$$

Depending on the signs of both invasion growth rates, we therefore have conditions for $\lambda_{1}^{2}$ and $\lambda_{2}^{1}$, leading to four ecological scenarios between two strains as in Madec and Gjini (2020) (see Table 2).

Table 2: System equilibria for 2-strain dynamics according to $\lambda_{1}^{2}$ and $\lambda_{2}^{1}$ as expected from the replicator equation 2.7

\begin{tabular}{llll}
\hline $\begin{array}{l}\text { Mutual invasion } \\
\left(\lambda_{1}^{2}, \lambda_{2}^{1}\right)\end{array}$ & Outcome & Strain frequencies & $\begin{array}{l}\text { Quadratic } \\
Q=z^{T} \Lambda z\end{array}$ \\
\hline$(+,+)$ & & form \\
$(-,+)$ & Stable coexistence & $z_{1}^{*}>0, z_{2}^{*}>0: \frac{1}{\lambda_{1}^{2}+\lambda_{2}^{1}}\left(\begin{array}{c}\lambda_{1}^{2} \\
\lambda_{2}^{1}\end{array}\right)$ & $Q>0$ and $Q \rightarrow Q^{*}$ \\
$(+,-)$ & Exclusion of type 1 & $z_{1}^{*}=0, z_{2}^{*}=1$ & $Q \rightarrow 0$ \\
$(-,-)$ & Exclusion of type 2 & $z_{1}^{*}=1, z_{2}^{*}=0$ & $Q \rightarrow 0$ \\
\hline
\end{tabular}

Thus, to investigate the equilibria and their stability in this 2-strain system, it suffices to study the values and signs of pairwise invasion fitness coefficients $\left(\lambda_{1}^{2}, \lambda_{2}^{1}\right)$, given explicitly as follows:

$$
\begin{aligned}
& \lambda_{1}^{2}=\theta_{1}\left(b_{1}-b_{2}\right)+\theta_{2}\left(\nu_{2}-\nu_{1}\right)+\theta_{3}\left(-u_{12}-u_{21}+2 u_{22}\right)+\theta_{4}\left(\omega_{12}^{1}-\omega_{21}^{2}\right)+\theta_{5}\left(\mu\left(\alpha_{21}-\alpha_{12}\right)+\alpha_{21}-\alpha_{22}\right) \\
& \lambda_{2}^{1}=-\theta_{1}\left(b_{1}-b_{2}\right)-\theta_{2}\left(\nu_{2}-\nu_{1}\right)+\theta_{3}\left(-u_{21}-u_{12}+2 u_{11}\right)-\theta_{4}\left(\omega_{12}^{1}-\omega_{21}^{2}\right)+\theta_{5}\left(\mu\left(\alpha_{12}-\alpha_{21}\right)+\alpha_{12}-\alpha_{11}\right),
\end{aligned}
$$

while their sum is $\lambda_{1}^{2}+\lambda_{2}^{1}=2 \theta_{3}\left(u_{11}+u_{22}-u_{12}-u_{21}\right)+\theta_{4}\left(\alpha_{12}+\alpha_{21}-\alpha_{11}-\alpha_{22}\right)$. It's not easy to determine the exact long time scenario or the winner in a two-strain system because parameters with perturbations affect all together the dynamics. The table 2 gives us criteria to determine the long time behavior. However, instead of computing the fitness coefficients explicitly in each case, we can base on 3.1 to determine quickly and for a various range of cases. Inspecting closely equations (3.1), we can see two parts: 
1. The part $\theta_{1} \Delta b+\theta_{2} \Delta \nu+\theta_{4} \Delta \omega+\theta_{5} \mu\left(\alpha_{21}-\alpha_{12}\right)$, which keeps the $\left(\lambda_{2}^{1}, \lambda_{1}^{2}\right)$ close to the line $\lambda_{1}^{2}+\lambda_{2}^{1}=0$, i.e. the dynamics tend to exclusion of one strain.

2. The part $\theta_{3} \Delta_{2} u+\theta_{5}\left(\alpha_{21}-\alpha_{22}\right)$, which pulls $\left(\lambda_{2}^{1}, \lambda_{1}^{2}\right)$ away from the line $\lambda_{1}^{2}+\lambda_{2}^{1}=0$, i.e. driving the dynamics toward coexistence or bi-stability.

This makes it easy to see that variation in transmissibility $(\Delta b)$ or duration of carriage between strains $(\Delta \nu)$, and the precedence effect in transmission from mixed coinfection $(\Delta \omega)$, always promotes competitive exclusion in the system, whereas variation in coinfection parameters (susceptibilities and clearance rates) can oppose competitive exclusion.

\subsection{An overview on four system outcomes dependent on $R_{0}$ and $k$}

\section{What determines competitive exclusion?}

The competitive exclusion occurs if and only if $\lambda_{1}^{2}>0$ and $\lambda_{2}^{1}<0$ or reversely, $\lambda_{1}^{2}<0$ and $\lambda_{2}^{1}>0$. By (3.1), taking $R_{0} \rightarrow 1^{+}$or $k \rightarrow 0^{+}$, which implies $\mu \rightarrow \infty$, makes the nonlinear part tends to 0 , which leads to the competitive exclusion. This remark coincides with the result about $\mu=\frac{1}{\left(R_{0}-1\right) k}$ in Gjini and Madec (2021a).

We note that a biologically feasible range for $R_{0}$ and $k$ is: $1 \leq R_{0} \leq 10$ and $0 \leq k \leq 10$, thus we use values of these parameters in such range to illustrate our model behavior through simulations. However, the model is general to accommodate any other positive values of such parameters.

Next, we will consider the case if we take $\mu \rightarrow \infty$, which implies $R_{0} \rightarrow 1^{+}$or $k \rightarrow 0^{+}$, and determine the strain winning in competitive exclusion.

As mentioned, we can rewrite pairwise invasion fitness so that we highlight two opposing terms, where the first one, is completely anti-symmetric in the reverse $\lambda_{2}^{1}$, thus contributes only to competitive exclusion. Whereas, the second term in the square bracket captures the trait variation that may lead to outcomes beyond exclusion. Here, recall that, if we impact on the system so that $R_{0} \rightarrow 1$ or $k \rightarrow 0$ to get the phenomenon of exclusion, then $\frac{\theta_{2}}{\theta_{1}} \rightarrow \frac{\gamma}{\gamma+r}$, and $\frac{\theta_{3}}{\theta_{1}}, \frac{\theta_{5}}{\theta_{1}}$ go to 0 . Hence, the second part tends to 0 and determination of winner/extinct strain depends on the sign of

$$
\theta_{1}\left(b_{1}-b_{2}\right)+\theta_{2}\left(\nu_{2}-\nu_{1}\right)+\theta_{4}\left(\omega_{i j}^{i}-\omega_{j i}^{j}\right)+\theta_{5} \mu\left(\alpha_{21}-\alpha_{12}\right) .
$$

If the sign of this expression is positive then strain 1 will be the winner strain and vice versa. Generally, using these arguments, it is still hard to consider exactly the single winner without computing explicitly the term (3.2). The final answer will depend on the advantage in terms including transmission $\Delta b$, duration of carriage $\Delta \nu$, transmission probability of a strain from a co-colonized host $\omega_{12}^{1}-\omega_{21}^{2}$ and susceptibility to co-colonization $\left(\alpha_{21}-\alpha_{12}\right)$. We will study particular cases in the next sections, but an overview of the range of possible scenarios is given in Table 3.

\section{What determines coexistence?}

By the previous arguments, in order to have the coexistence of two strains, in our model with coinfection, the essential condition is $R_{0}>1$ and $k>0$ large enough. Coexistence opportunities can only come from advantages that may arise in coinfection. In other words, this requires the ratio of single to co-colonization $\mu$ tend to 0 . In conclusion, from the analysis until here, we have that:

1. The perturbations only in $\beta_{i}, \gamma_{i}$ and $p_{i j}^{i}$ lead to the competitive exclusion. Thus strain-specific transmission and/or clearance rates, and the strain-specific transmission biases from mixed co-colonized hosts only create forces favouring exclusion in the system.

2. The perturbations in co-colonization clearance rates and susceptibilities, $\gamma_{i j}$ and $k_{i j}$, create more complex scenarios including exclusion of each strain, coexistence or bistable exclusion steady states. Thus, only through the possibility of asymmetries in co-colonization (co-infection) parameters can the strains mediate their mutual coexistence.

In the next section, we will consider the phenomena: exclusion, coexistence or bistable exclusion of either strain according to $\mu$. 
Table 3: Which trait variation between 2 strains leads to which final outcome? Scenarios of variation in biological parameters analyzed with the quasi-neutral coinfection SIS model for 2-strains, and the final ecological outcome of their dynamics. There can be exclusion of strain 1 (E1), exclusion of strain 2 (E2), coexistence (C), and bistability (B). Not all trait variations lead to coexistence. In a majority of scenarios, for a given set of trait variation on $\geq 2$ trait axes, the final outcome between two strains may shift with coinfection prevalence in the system.

\begin{tabular}{|c|c|c|c|c|c|c|}
\hline Ecology & \multicolumn{3}{|c|}{ Exclusion Axis } & \multicolumn{2}{|c|}{ Coexistence-Bistability Axis } & \\
\hline $\begin{array}{l}\text { Nr. } \\
\text { traits } \\
\text { varying }\end{array}$ & $\beta_{i}$ & $\gamma_{i}$ & $p_{i j}^{i}$ & $\gamma_{i j}$ & $k_{i j}$ & Final outcome - Equilibrium \\
\hline 1 & $\checkmark$ & $x$ & $x$ & $x$ & $x$ & E1 or E2 (depends on $\left.R_{0}^{i}\right)$ \\
\hline 1 & $x$ & $\checkmark$ & $x$ & $x$ & $x$ & E1 or E2 (depends on $R_{0}^{i}$ ) \\
\hline 1 & $x$ & $x$ & $\checkmark$ & $\times$ & $x$ & E1 or E2 (depends on $\left.\omega_{12}^{1}-\omega_{21}^{2}\right)$ \\
\hline 1 & $x$ & $x$ & $x$ & $\checkmark$ & $x$ & E1, E2, C, B (depends on $u_{i j}$ ) \\
\hline 1 & $x$ & $x$ & $x$ & $x$ & $\checkmark$ & $\mathrm{E} 1, \mathrm{E} 2, \mathrm{C}, \mathrm{B}$ (depends on $\alpha_{i j},(* *)$ \\
\hline 2 & $\bullet$ & $\bullet$ & $\bullet$ & $x$ & $x$ & $\mathrm{E} 1$ or $\mathrm{E} 2(*)$ \\
\hline 3 & $\checkmark$ & $\checkmark$ & $\checkmark$ & $x$ & $x$ & $\mathrm{E} 1$ or $\mathrm{E} 2(* *)$ \\
\hline$\geq 2$ & $\bullet$ & $\bullet$ & $\bullet$ & $\checkmark$ & $x$ & $\mathrm{E} 1, \mathrm{E} 2, \mathrm{C}, \mathrm{B}(\#)$ \\
\hline$\geq 2$ & $\bullet$ & $\bullet$ & $\bullet$ & $\times$ & $\checkmark$ & $\mathrm{E} 1, \mathrm{E} 2, \mathrm{C}, \mathrm{B}(\#)$ \\
\hline$\geq 2$ & $\bullet$ & $\bullet$ & $\bullet$ & $\checkmark$ & $V$ & $\mathrm{E} 1, \mathrm{E} 2, \mathrm{C}, \mathrm{B}(\#)$ \\
\hline
\end{tabular}

The role of co-colonization (co-infection) as a potential gradient for system behavior:

$\left(^{*}\right)$ In this case, for any combination of fixed trait variation between two strains (with traits denoted by $\bullet$ varying or not varying), there is only competitive exclusion and up to one shift is possible as a function of $\mu\left(I^{*} / D^{*}\right)$. Thus the system can shift from Exclusion of $1 \rightarrow$ Exclusion of 2 or vice versa, only once, because of a particular overall coinfection prevalence $(D / T=1 /(\mu+1))$.

(**) In this case, for any combination of fixed trait variation between two strains, only competitive exclusion is possible, but now up to 2 shifts are possible as a function of $\mu\left(I^{*} / D^{*}\right)$. Thus the system can shift three times between opposite exclusion equilibria, because of overall coinfection prevalence.

(\#) In all these cases, (with traits denoted by $\bullet$ varying or not varying), for any combination of fixed trait variation between two strains, there can be more qualitative shifts between scenarios as a function of $\mu\left(I^{*} / D^{*}\right.$, i.e. $R_{0}$ and $\left.k\right)$. For example, by varying coinfection prevalence, the system can shift: Exclusion $\rightarrow$ Coexistence $\rightarrow$ Exclusion; or Exclusion $\rightarrow$ Bistability $\rightarrow$ Exclusion (see Figs. S3 S4). In very specific cases, for a given combination of fixed trait variation between two strains, the system may even shift 4 times to different regimes as a function of $\mu$, depending on how the $\lambda_{1}^{2}$ and $\lambda_{2}^{1}$ intersect the $\mathrm{x}$-axis and each-other (see Supplementary proofs in Text S3.

\section{Effect on each trait variation on final outcome}

In this very general version of the model, two strains vary along several fitness dimensions: transmission, clearance rate, co-colonization interactions, and possible biases in the clearance of co-colonization and transmission from the co-colonization compartment. In the following we will explore these dimensions in detail, and what is their effect on the competitive dynamics for $N=2$.

In the subsections 4.1, 4.2, 4.3, and 4.4, for each case considered, we study the values of mutual invasion fitnesses $\lambda_{1}^{2}$ and $\lambda_{2}^{1}$ as a function of the ratio of single to co-colonization $\mu$. Without loss of generality, in computation we assume that $\nu_{1}<\nu_{2}$, hence strain 1 is cleared more slowly than strain 2 , giving it an advantage in duration of carriage $(\Delta \nu>0)$. First, we recall the relative weights $\left(\theta_{i}\right)$ of each trait in terms of $\mu, \mu \in[0,+\infty)$ in the Table 1 .

\subsection{Definite drivers of competitive exclusion}

In this model, exclusion always results from strain-specific transmission and clearance rate of single infection, and transmission probability from coinfected hosts, if other parameters are equal. Below we explore these three axes of trait variation in more detail. First, we consider variation only in $\beta_{i}, \gamma_{i}$ and $p_{i j}^{i}$. We assume equal parameters for co-colonization clearance $\gamma_{i j}=\gamma$ and interaction coefficients between strains $k_{i j}=k$. The two invasion fitnesses (Figure 2) are:

$$
\left\{\begin{array}{l}
\lambda_{1}^{2}=\frac{\gamma}{2(\gamma+r)} \mu(2 \mu+1) \Delta \nu+(\mu+1)^{2} \Delta b+(\mu+1) \Delta \omega \\
\lambda_{2}^{1}=-\lambda_{1}^{2}
\end{array}\right.
$$



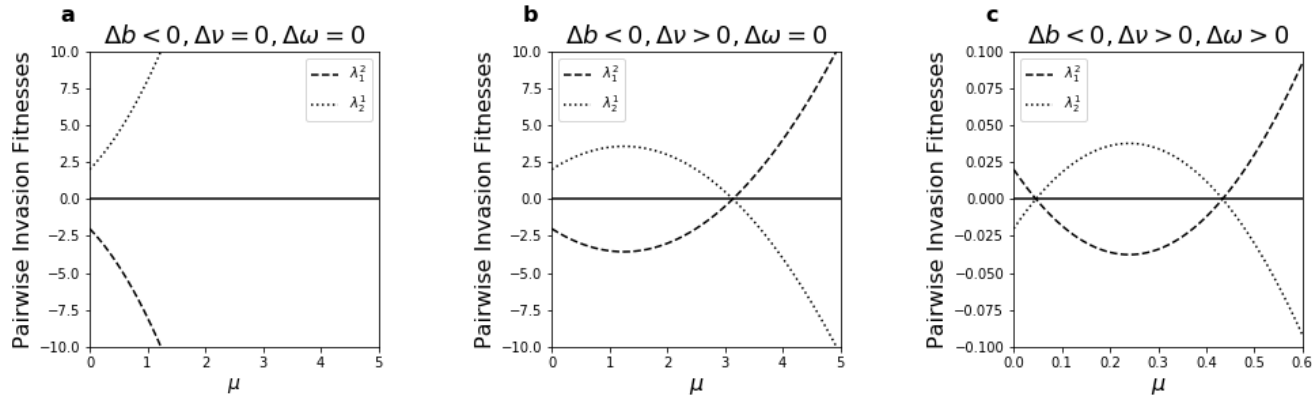

Figure 2: Pure competitive exclusion from variation in three traits, but the winner strain may depend on $\mu$. Competitive exclusion is the only scenario when two strains vary only in transmission rate $\beta_{i}$ and/or infection clearance rate $\gamma_{i}$ and/or transmission probability from coinfected hosts $p_{i j}^{i}$ (See (4.1)). Completely anti-symmetric mutual invasion leads to competitive exclusion. The strain with the positive invasion fitness excludes the one with the negative invasion fitness. Here we illustrate the values of $\lambda_{1}^{2}$ and $\lambda_{2}^{1}$ as a function of the ratio of single to co-colonization in the system $\mu=1 /\left(k\left(R_{0}-1\right)\right)$, with $\gamma=1.5, r=0.5$, in three cases of (a) Variations in transmission rates $\beta_{i}$ only with $\Delta b=-2$, (b) Variations in transmission rates $\beta_{i}$ and clearance rates $\gamma_{i}$ with $\Delta \nu=4$ and $\Delta b=-2$, and $(\mathbf{c})$ Variations in transmission rates $\beta_{i}$, clearance rates $\gamma_{i}$ and transmission probability from coinfected hosts $p_{i j}^{i}$ with $\Delta \nu=4, \Delta b=-2$ and $\Delta \omega=2.02$.

These fitness coefficients are completely anti-symmetric, implying competitive exclusion as the only outcome. Thus it doesn't matter that there is co-colonization in the system $(k>0)$. For promoting coexistence, this is not sufficient by itself. Variable co-colonization interactions or traits between strains would be an additional requirement. When strains behave equally in all processes related to coinfection, coinfection cannot rescue them from the destiny of competitive exclusion. However, as we explore below, overall prevalence of coinfection can actually shift between the winning and losing strain. This can happen only if there is variation in duration of carriage. For example noticing that when $\beta_{i}$ is the only trait varying between two strains, $k$ does not appear in $\theta_{1}$, this indicates that transmissibility's variation, uniquely determines the winner between two strains; its relative contribution to $\lambda_{i}^{j}$ cannot be altered by coinfection.

If variation is only in transmission rates $\beta_{i}$, then the strain with bigger $\beta_{i}$ excludes the other one, see figure 2 (b). This fact holds for $N$ strains in general and is proved in Le et al. (2021).

If a strain is superior in both fitness dimensions, which means it has smaller clearance rate and greater transmission rate $(\Delta b>0, \Delta \nu>0)$, then for all value of $\mu$ or $R_{0}$, it surely will be the winner par excellence, which can be easily seen from (4.1). This is unsurprising and naturally expected.

However, if a strain is better in one trait but worse in another, for example if the strain with longer duration of carriage (lower clearance) also has smaller transmission rate, the determination of the winning strain depends on value of $\mu$ (and in general also $R_{0}$ ), see Figure $2 \mathrm{~b}$.

Since we have fixed here $\gamma=1.5, r=0.5$ by convention, if we fix neutral transmission rate $\beta$, the value of $\mu$ now depends only on the mean interaction coefficient in co-colonization $k$. This means, when $k$ is high, thus when strains tend to allow each-other more in co-colonization, $\mu$ is sufficiently close to 0 , strain 2 , which has the smaller transmission rate but longer duration of carriage is the winner. In contrast, when $\mu$ is larger, thus when hosts are less vulnerable to co-colonization, the strain 1, which has bigger transmission rate and smaller duration of carriage is the winner.

This illustrates how the relative advantage between two strains, differing in two traits, depends on coinfection prevalence.

It is interesting to note that, if variations are only in two of which including transmission rates $\beta_{i}$, clearance rates $\gamma_{i}$ and transmission probability from coinfected hosts $p_{i j}^{i}$, we can have at most one shifting outcome, i.e. shifting once from the exclusion of one strain to the exclusion of the other one, see proof in $S 3.1$.

Following the same analysis, we can study the model in which transmission probability from co-colonized hosts, denoting within-host advantage, $p_{i j}^{i}$ displays strain-specific perturbation. We note that if there is variation in $p_{i j}^{i}$ only, if $\omega_{12}^{1}-\omega_{21}^{2}>0$, strain 1 excludes strain 2 , and vice versa. This can be understood via the precedence advantage that one strain has from mixed coinfected hosts if it arrives first, and thus gets transmitted more.

If there are combinations of variations in within-host transmission advantage $p_{i j}^{i}$, as well as other traits $\beta_{i}$ and/or $\gamma_{i}$, the final outcome is more complex (see Table/3 and Figure 2(c). However, the long time competitive result is always exclusion of one strain from the system. If perturbations occur in transmission rate $\beta_{i}$ and transmission probabilities from mixed coinfection $p_{i j}^{i}$, the winning strain depends on coinfection prevalence in the system, $\left(\mu=I^{*} / D^{*}\right)$ because $\frac{\theta_{4}}{\theta_{1}}=\frac{1}{1+\mu}$. If perturbations occur in duration of single carriage (i.e. 
strain-specific clearance rates $\gamma_{i}$ ) and $p_{i j}^{i}$, the winning strain depends on more strain-transcending parameters: $\mu, \gamma$ and $r$. This can be explicitly observed in the ratio $\frac{\theta_{4}}{\theta_{2}}=\frac{2(\gamma+r)}{\gamma} \frac{\mu+1}{\mu(2 \mu+1)}$, which ultimately affects the signs of pairwise invasion fitnesses. Figure 2 (c shows us a special example in which the exclusion of either strains shifts twice when $\mu$ varies from 0 to $\infty$.

\subsection{Four scenarios possible with variable co-infection clearance rates $\gamma_{i j}$}

As we can see from the fully explicit expression of pairwise invasion fitness, in this model, the coinfection clearance rate axis contributes to $\lambda_{i}^{j}$ with a term $2\left(u_{j j}-\frac{u_{j i}+u_{i j}}{2}\right)$. Thus what matters is the comparison between clearance rate of same strain coinfection vs. the mean coinfection clearance rate of mixed-strain coinfection. While there are no restrictions for how these can vary, in the following we consider three special cases where the variation in coinfection duration depends on variation in single infection duration. We also assume no variation in transmission probability from coinfected hosts $\left(p_{i j}^{i}=1 / 2\right)$ but the results for the general case can be easily derived using Eqs. (3.1).

\subsubsection{Case 1: Unbiased clearance in mixed carriage $u_{12}=u_{21}=\frac{\nu_{1}+\nu_{2}}{2}$}

In this first case, we consider that the clearance rate of mixed carriage is unbiased and equal to the mean of the two clearance rates of single colonization. We have $u_{11}=\nu_{1}, u_{22}=\nu_{2}$ and the invasion fitnesses between two strains are anti-symmetric:

$$
\left\{\begin{array}{l}
\lambda_{1}^{2}=\frac{\gamma}{2(\gamma+r)}\left(2 \mu^{2}+2 \mu+1\right) \Delta \nu+(1+\mu)^{2} \Delta b \\
\lambda_{2}^{1}=-\lambda_{1}^{2}
\end{array}\right.
$$

This case leads again to the pure competitive exclusion. Whichever strain has positive $\lambda_{i}^{j}$ will be the winner.

- Similar to the case in section 3.1, if a strain is superior in both transmission $\beta_{i}$ and clearance $\nu_{i}$, it will be the winner.

- However, if $\Delta \nu$ and $\Delta b$ have opposite sign, meaning one strain has advantage in clearance and the other has advantage in transmission, the final winner will depend on coinfection prevalence, hence on $\mu$. From the formula of the invasion fitness, it can be seen that in this system, the clearance rate differential (i.e. in duration of carriage) has more important role than the transmission rate difference in helping an inferior strain overcome and overturn its fitness disadvantage as $\mu$ increases.

\subsubsection{Case 2: Decreased clearance in mixed carriage $u_{12}=u_{21}=\min \left\{\nu_{1}, \nu_{2}\right\}=\nu_{1}$}

Here, we explore the case when mixed carriage clearance rate corresponds to the minimum of the two single colonization clearance rates. We still have $u_{11}=\nu_{1}, u_{22}=\nu_{2}$, but in hosts carrying a mixture of strain 1 and strain 2, this creates an advantage in co-colonization for the opponent strain (the one with the faster strain-specific clearance). The two invasion fitness coefficients are not anti-symmetric anymore, hence allowing for more scenarios beyond exclusion (see figures $3(\mathbf{a}, \mathbf{b})$ ):

$$
\begin{array}{ll}
\lambda_{1}^{2}=\frac{\gamma}{2(\gamma+r)}[(2 \mu+1) \mu+2(\mu+1)] \Delta \nu & +(\mu+1)^{2} \Delta b \\
\lambda_{2}^{1}=-\frac{\gamma}{2(\gamma+r)}(2 \mu+1) \mu \Delta \nu & -(\mu+1)^{2} \Delta b .
\end{array}
$$

- If one strain, denoted to be strain 1 in Figure 3 a, has larger transmission rate and lower clearance rate (case when $\Delta b>0, \Delta \nu>0$ ), it will again be the only survivor for all $\mu$.

- However if the advantage is only in one of the two traits (case when $\Delta b, \Delta \nu$ have opposite signs), for example the strain with smaller clearance rate has lower transmission rate, as in Figure $3 \mathrm{~b}$, then coexistence can occur. However, even in this situation, coexistence can only be possible for sufficiently low $\mu$, i.e. $k$ large enough for fixed $R_{0}$ (we already fix $\gamma$ and $r$ ). This means that increasing the relative prevalence of co-colonization or coinfection in the system, via higher facilitative interactions, can promote coexistence of two strains. This links back to the arguments at the end of section 2 .

The phenomenon of coexistence arising here is similar to what has been found before in the context of virulence evolution (Alizon, 2008), where decreased clearance in coinfection was observed to promote coexistence, hence persistence of more virulent strains (here strain 2 if $\Delta \nu>0$, and in the limit of strain $2-R_{0}$ below 1 . 

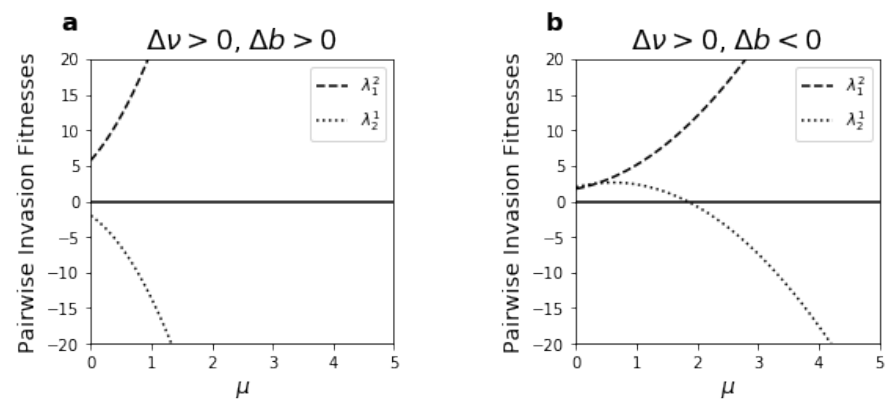

Case $2: u_{i j}=\min \left\{v_{i}, v_{j}\right\}$
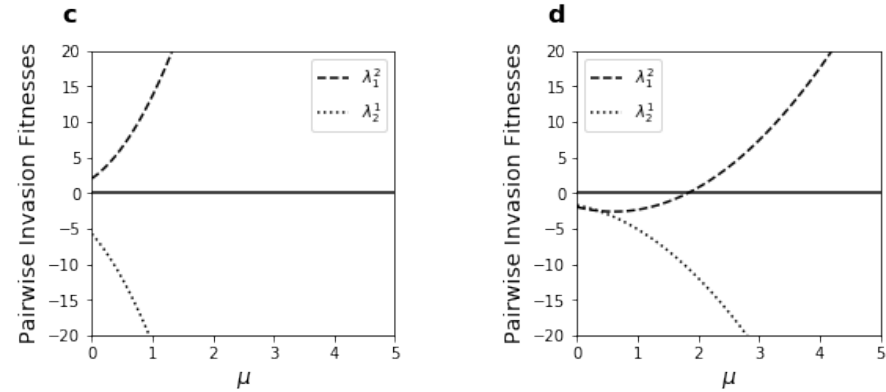

Case 3: $u_{i j}=\max \left\{v_{i}, v_{j}\right\}$

Figure 3: Competitive exclusion can be broken with variable coinfection clearance rates, and the result may depend on $\mu$. We illustrate possible scenarios resulting from deviation from symmetry in the mixed coinfection clearance rate $\gamma_{i j}$ as a function of the ratio of single to coinfection $\mu$. a-b Coinfection clearance equals the minimum clearance rate of either strain: $u_{12}=u_{21}=\min \left\{\nu_{1}, \nu_{2}\right\}=\nu_{1}$ (see (4.3)). c-d Coinfection clearance equals the maximum clearance rate of either strain: $u_{12}=u_{21}=\max \left\{\nu_{1}, \nu_{2}\right\}=\nu_{2}$ (see (4.4)). We choose $\gamma=1.5$ and $r=0.5$ as in figure 2 For each sub case, we plot the mutual invasion fitnesses for transmission advantage and disadvantage of strain 1 , respectively: $\Delta b>0(\mathbf{a}, \mathbf{c})$ and $\Delta b<0(\mathbf{b}, \mathbf{d})$. In particular, in the first column $\Delta b=2$, and in the second column $\Delta b=-2$. The clearance rate differential $\Delta \nu$ is assumed $\Delta \nu=5$ attributing higher duration of carriage to strain 1 . In the first column, strain 1 is superior in all fitness dimensions, and coinfection clearance cannot overturn the result. In the second column, strain 1 is not superior in all fitness dimensions, and coinfection matters for the final result.

\subsubsection{Case 3: Increased clearance in mixed carriage $u_{12}=u_{21}=\max \left\{\nu_{1}, \nu_{2}\right\}=\nu_{2}$}

The co-infection clearance rate in mixed carriage here is assumed to be equal to the maximum value of the strain-specific clearance rates in single colonization. We have $u_{11}=\nu_{1}, u_{22}=\nu_{2}$ and the pairwise invasion fitness coefficients are (see figures $3(\mathbf{c}, \mathbf{d}))$ :

$$
\begin{array}{ll}
\lambda_{1}^{2}=\frac{\gamma}{2(\gamma+r)}(2 \mu+1) \mu \Delta \nu & +(\mu+1)^{2} \Delta b \\
\lambda_{2}^{1}=-\frac{\gamma}{2(\gamma+r)}[(2 \mu+1) \mu+2(\mu+1)] \Delta \nu & -(\mu+1)^{2} \Delta b
\end{array}
$$

- If strain 1 has smaller clearance rate and larger transmission rate, it is again the superior strain in the system, independently of coinfection parameters, like previous cases (Figure 3r).

- However, if strain 1 has smaller clearance rate but also lower transmission rate, bistability of exclusion can occur when $\mu$ is small enough. When $\mu$ becomes larger and tends to infinity, we obtain only competitive exclusion, as mentioned earlier and proven in Section 2. In that extreme, strain 1 is the only persistent strain over long time (Figure $3 \mathrm{~d})$.

In conclusion, by the explicit formulae of $\left(\lambda_{1}^{2}, \lambda_{2}^{1}\right)$ in the cases above, we can also prove that for $\mu$ large enough, the strain which has smaller clearance rate will be the only strain persisting in the system.

\subsection{Four scenarios from variation in pairwise co-colonization susceptibilities $k_{i j}$}

Another fitness dimension is how the strains facilitate or compete in altered susceptibilities to co-colonization via the coefficients $k_{i j}$. Above we assumed they are all equal to the reference $k$. But when variation in this parameter is allowed, as shown already in (Gjini and Madec, 2017), all four ecological scenarios are possible, and thus the effect is to open up space for coexistence and bistability among two strains, when competitive exclusion is expected from other parameters. According to the derivation of the reduced model in Madec and Gjini (2020), the perturbations in co-colonization interaction matrix for $N=2$ satisfy $\sum_{i, j=1}^{2} \alpha_{i j}=0$ when $k$ is defined by the mean of $k_{i j}$. However, without loss of generality, one can shift the $\alpha_{i j}$ by the same constant, 
without changing the mutual $\lambda_{i}^{j}$ and consequently without changing the dynamics. The explicit formulas for two pairwise invasion fitnesses are (see figure 4)

$$
\begin{aligned}
& \lambda_{1}^{2}=\frac{\gamma}{2(\gamma+r)}(2 \mu+1) \mu \Delta \nu+\left(R_{0}-1\right) \mu\left(\mu\left(\alpha_{21}-\alpha_{12}\right)+\alpha_{21}-\alpha_{22}\right)+(\mu+1)^{2} \Delta b, \\
& \lambda_{2}^{1}=-\frac{\gamma}{2(\gamma+r)}(2 \mu+1) \mu \Delta \nu+\left(R_{0}-1\right) \mu\left(\mu\left(\alpha_{12}-\alpha_{21}\right)+\alpha_{12}-\alpha_{11}\right)-(\mu+1)^{2} \Delta b .
\end{aligned}
$$

In this spirit, below we consider a few special cases of $\alpha_{i j}$ variation between strains, to highlight the effect of co-colonization susceptibilities when they provide:

i) a disadvantage to strain 1: $\left(\alpha_{i j}\right)_{i j}=\left(\begin{array}{cc}-1.5 & 0.5 \\ 0.5 & 0.5\end{array}\right)$, whose effect on $\lambda_{i}^{j}$ is equivalent to $\left(\alpha_{i j}\right)_{i j}=$ $\left(\begin{array}{cc}-2 & 0 \\ 0 & 0\end{array}\right)$, and increases relatively $\lambda_{2}^{1}$ :

$$
\left\{\begin{array}{l}
\lambda_{1}^{2}=\frac{\gamma}{2(\gamma+r)}(2 \mu+1) \mu \Delta \nu+(\mu+1)^{2} \Delta b \\
\lambda_{2}^{1}=-\frac{\gamma}{2(\gamma+r)}(2 \mu+1) \mu \Delta \nu+2\left(R_{0}-1\right) \mu-(\mu+1)^{2} \Delta b
\end{array}\right.
$$

ii) an advantage to strain 2: $\left(\alpha_{i j}\right)_{i j}=\left(\begin{array}{cc}-0.5 & -0.5 \\ -0.5 & 1.5\end{array}\right)$; equivalent to $\left(\alpha_{i j}\right)_{i j}=\left(\begin{array}{cc}0 & 0 \\ 0 & 2\end{array}\right)$ when shifted by the appropriate constant, which relatively decreases $\lambda_{1}^{2}$ :

$$
\left\{\begin{array}{l}
\lambda_{1}^{2}=\frac{\gamma}{2(\gamma+r)}(2 \mu+1) \mu \Delta \nu-2\left(R_{0}-1\right) \mu+(\mu+1)^{2} \Delta b, \\
\lambda_{2}^{1}=-\frac{\gamma}{2(\gamma+r)}(2 \mu+1) \mu \Delta \nu-(\mu+1)^{2} \Delta b .
\end{array}\right.
$$

iii) and exactly counterbalanced effects on either strain: $\left(\alpha_{i j}\right)_{i j}=\left(\begin{array}{cc}-\sqrt{2} & 0 \\ 0 & \sqrt{2}\end{array}\right)$, whose impact on $\lambda_{i}^{j}$ is to decrease $\lambda_{1}^{2}$ and increase $\lambda_{2}^{1}$ by exactly the same amount:

$$
\left\{\begin{array}{l}
\lambda_{1}^{2}=\frac{\gamma}{2(\gamma+r)}(2 \mu+1) \mu \Delta \nu-\sqrt{2}\left(R_{0}-1\right) \mu+(\mu+1)^{2} \Delta b, \\
\lambda_{2}^{1}=-\frac{\gamma}{2(\gamma+r)}(2 \mu+1) \mu \Delta \nu+\sqrt{2}\left(R_{0}-1\right) \mu-(\mu+1)^{2} \Delta b .
\end{array}\right.
$$

In Figure 4, we consider these cases, where besides $k_{i j}$, we allow also variation in transmission $\beta$ and clearance $\gamma$ of each strain, but assume initially symmetry in other traits. We assume $\Delta \nu>0$ (strain 1 is cleared more slowly). In Fig. 4 -c we illustrate competitive outcomes (dependent on mutual signs of $\lambda_{i}^{j}$ ), when strain 1 is superior in transmissibility, and in Fig. 4 $\mathrm{d}-\mathrm{f}$ we show outcomes when strain 2 is superior in transmissibility instead. Naturally as $\mu \rightarrow \infty$ the role of coinfection interaction asymmetries vanishes, and the system tends to exclusion, but for low values of $\mu$, the structure of the $\alpha_{i j}$ matters. In particular, if it is asymmetric (Figure $4 \mathrm{a}-\mathrm{b}, \mathrm{d}-\mathrm{e}$ ) there can be at most 3 scenarios as a function of $\mu$ : exclusion - coexistence -exclusion, or exclusion - bistability- exclusion. In particular increase in $\lambda_{1}^{2}$ acts to enable coexistence when $\Delta b>0$, and a decrease in $\lambda_{2}^{1}$ acts to enable bistability when $\Delta b<0$. Whereas, if co-colonization interactions have exactly counterbalanced effects on $\lambda_{i}^{j}$ (Fig. 4 f,f), there can only be alternating exclusion scenarios as a function of $\mu$.

\subsection{Adding variation in transmission probability from coinfected hosts}

In this case, besides transmission and clearance rates variations $(\Delta b, \Delta \nu)$, and co-colonization susceptibilities $\left(\alpha_{i j}\right)$, we add to the system variation in terms of a slight priority effect between strains for transmission from coinfection $\Delta \omega \neq 0$. The explicit formulae for pairwise invasion fitnesses of two strains are as follows:

$$
\begin{aligned}
& \lambda_{1}^{2}=\frac{\gamma}{2(\gamma+r)}(2 \mu+1) \mu \Delta \nu+(\mu+1) \Delta \omega+\left(R_{0}-1\right) \mu\left(\mu\left(\alpha_{21}-\alpha_{12}\right)+\alpha_{21}-\alpha_{22}\right)+(\mu+1)^{2} \Delta b \\
& \lambda_{2}^{1}=\frac{\gamma}{2(\gamma+r)}(2 \mu+1) \mu \Delta \nu-(\mu+1) \Delta \omega+\left(R_{0}-1\right) \mu\left(\mu\left(\alpha_{12}-\alpha_{21}\right)+\alpha_{12}-\alpha_{11}\right)-(\mu+1)^{2} \Delta b
\end{aligned}
$$

Recall that $\Delta \omega=\omega_{12}^{1}-\omega_{21}^{2}$ represents the relative advantage of strain 1 from arriving first within-host, in transmission from mixed coinfection. It is clear from the expression above, that when variation in this withinhost advantage is combined with variation in coinfection clearance rates or co-colonization susceptibility factors 

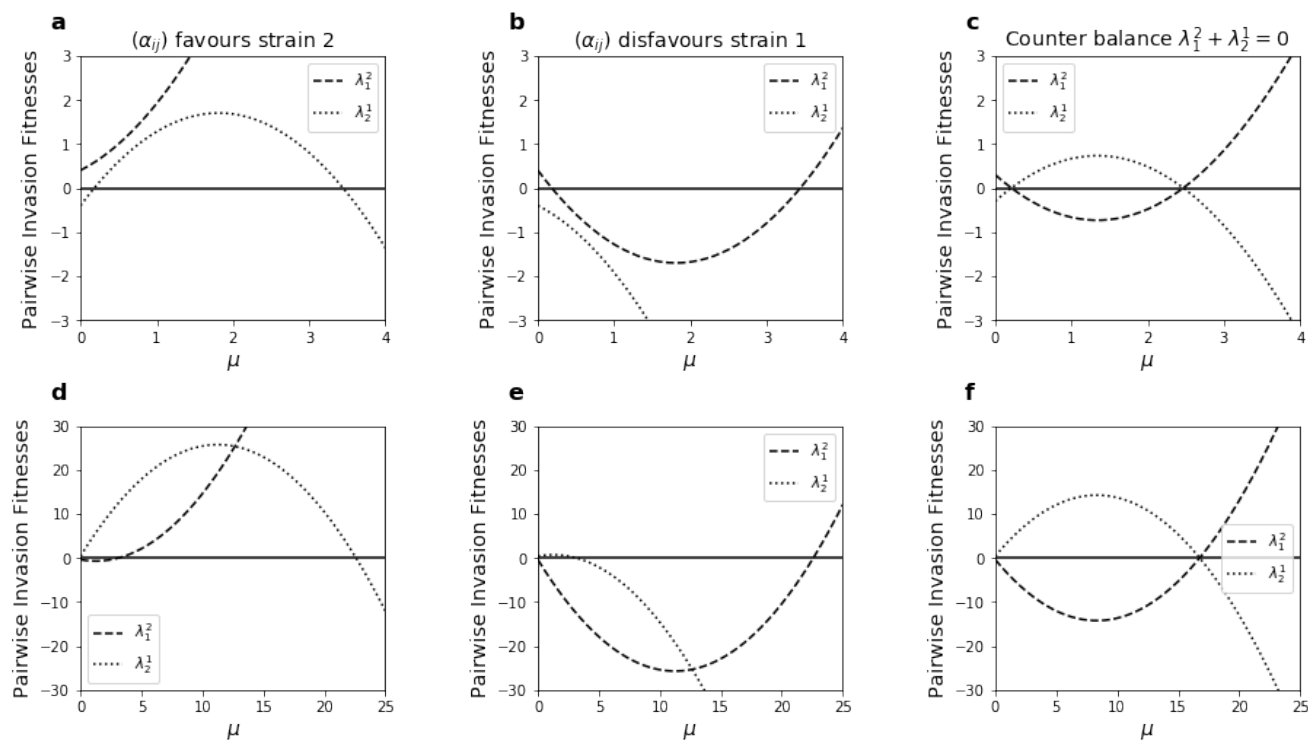

$\Delta b>0, \Delta v>0$

Figure 4: Breaking the competitive exclusion with co-colonization interactions $k_{i j}$ (see Eqs 4.5)). We compute pairwise invasion fitnesses $\left(\lambda_{1}^{2}, \lambda_{2}^{1}\right)$ according to $\mu$ in various cases of co-colonization interaction matrix $\left(\alpha_{i j}\right)$ with $R_{0}=5, r=0.5$ and $\gamma=1.5$. (a-c) We illustrate the cases of transmission superiority of strain $1: \Delta b>0$, when $\Delta b=0.4, \Delta \nu=0.8$. In (d-f) we plot 2-strain invasion fitnesses for transmission superiority of strain $2: \Delta b<0$, when $\Delta b=-0.4, \Delta \nu=0.8$, with the same $\gamma, r$ and $R_{0}$ as in $(\mathbf{a}, \mathbf{b}, \mathbf{c})$. Coinfection clearance rate $\gamma_{i j}$ is assumed equal to $\gamma$ and transmission probability from coinfected hosts carrying a mixture of two strains $p_{i j}^{i}=\frac{1}{2}$. Subplots with the same values $\left(\alpha_{i j}\right)$ lie in the same column. In particular, we consider 3 structures: $(\mathbf{a}, \mathbf{d})\left(\begin{array}{cc}-2 & 0 \\ 0 & 0\end{array}\right)($ Eqs 4.6$)$; (b, e) $\left(\begin{array}{ll}0 & 0 \\ 0 & 2\end{array}\right)($ Eqs 4.7$)$ ); (c, f) $\left(\begin{array}{cc}-\sqrt{2} & 0 \\ 0 & \sqrt{2}\end{array}\right)$ (Eqs 4.8) for variation in co-colonization interactions. Except for when the $\alpha_{i j}$ exactly counterbalance effects on $\lambda_{i}^{j}(\mathrm{c}, \mathrm{f})$ there is potential for more scenarios beyond competitive exclusion, induced by coinfection susceptibilities between strains.

between strains, coexistence and bistability also become possible. In this case within-host and between-host competition combine to give rise to different outcomes. In figure S6 we illustrate the effect of $\Delta \omega$ on the baseline outcomes of Figure 4 a-c. The effect of $\Delta w<0$ is to increase the potential for strain 2-only competitive exclusion and coexistence with strain 1 in the system, oftentimes overturning the baseline result especially so if $\mu$ small. The importance of transmission biases from mixed coinfection $\Delta w$ is unsurprisingly higher when relative coinfection prevalence is higher in the system.

\subsection{The qualitative outcome for two strains can shift multiple times with $\mu$}

Until now we have seen the important and explicit role of strain-transcending parameters, (e.g. $R_{0}, k$, and specifically $\mu$ ) which define the core neutral system of this model, on the ultimate competitive outcome between strains at the epidemiological level. We have seen that the same relative variation between strains, displayed in $(\Delta b, \Delta \nu, \ldots)$ will have a different impact in a system with larger or lower overall prevalence of coinfection, relationships that are completely transparent in the $\lambda_{i}^{j}$. Sometimes the effect will be quantitative, changing only the speed of dynamics without affecting the $\lambda_{i}^{j}$ signs (see Supplementary figure S2). Other times the effect will be qualitative, changing the signs of the pairwise invasion fitnesses and hence the dynamics. Furthermore, using such full analytic transparency, we can also prove mathematically special results to make the claims about qualitative shifts more precise. Depending on how many traits and which traits vary in the system, we can have at most one, two, or more shifts with $\mu$ (see Table 2). For example we can prove that with variation only in transmission and clearance rates, there can be at most one shift in final outcome as a function of $\mu$ (Text S2.1)

A very special case arises, when the same system can shift 4 times as a function of $\mu$. We have proven that a necessary condition for its occurrence is the presence of variation in both coinfection clearance rates $\gamma_{i j}$ and vulnerabilities to coinfection $k_{i j}$. If any of these is missing, 4 shifts as a function of $\mu$ are impossible (see Text S2.2 for the formal proof). An illustration of such a special case is given in Figure 5, where the system traces all 4 quadrants as $\mu$ goes from 0 to $\infty$, highlighting an extreme case of the critical role of coinfection, for the relative hierarchical advantages between two strains and their selection dynamics. We see that the system is characterized by competitive exclusion of strain 2 for $\mu$ low, then tends to coexistence of both strains 
for increasing $\mu$, followed by competitive exclusion of strain 1 for even higher $\mu$, until it returns again in the competitive exclusion region of strain 2 as $\mu \rightarrow \infty$.

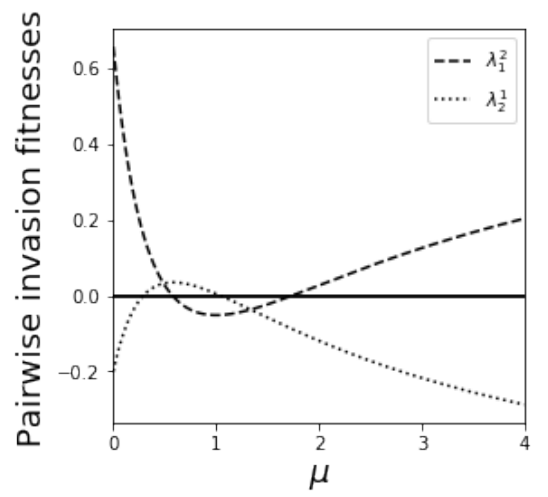

b

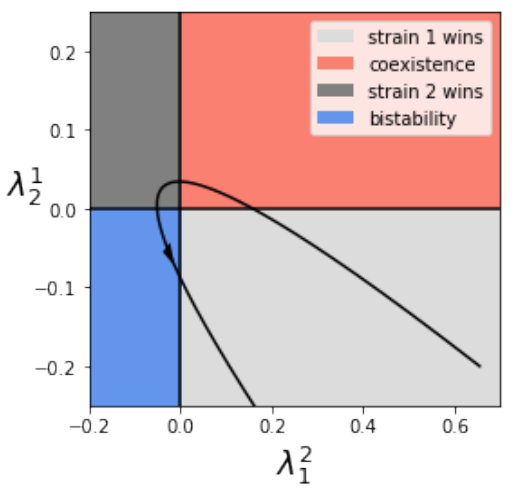

Figure 5: Four ecological scenarios may happen depending on $\mu$ under fixed trait variation. Variations here are in transmission rates, infection and coinfection clearance rates, and co-colonization susceptibilities between strains. The strain-transcending parameters are assumed $\gamma=2, r=0.2$ and $R_{0}=2.5$. We assume that $\Delta b=0.2, \Delta \nu=0.5$, the coinfection clearance rate $\gamma_{i j}$ with $u_{i j}=\min \left\{\nu_{i}, \nu_{j}\right\}$ and values of $\left(\alpha_{i j}\right)$ to be $\left(\begin{array}{cc}-1 & 0 \\ 0 & \sqrt{3}\end{array}\right)$. The ultimate ecological outcomes when $\mu$ goes from 0 to $\infty$ include: exclusion of strain 2 , coexistence, exclusion of strain 1 , bistablity, then back to the exclusion of strain 2. Figure (a) plots two pairwise invasion fitnesses as function of $\mu$. Figure (b) plots $\lambda_{1}^{2}$ against $\lambda_{2}^{1}$ as parametric functions of $\mu$ crossing all four quadrants.

\subsection{Parameter regions for four ecological outcomes in co-colonization}

Until now, we have considered fixed trait variations between two strains, and varied $\mu$ to show how their net competitive dynamics driven by $\lambda_{1}^{2}$ and $\lambda_{2}^{1}$ will depend on the ratio of single to coinfection in the neutral system. This context is defined by basic reproduction number $R_{0}$, and $k$, of the neutral model (see Table 1 ). Next, we consider the distribution of 4 possible ecological outcomes across different systems, as a function of $R_{0}$ and $k$. In figure 6 we represent the long time behaviour of a 2-strain model with perturbations in transmission rate $\beta_{i}$, clearance rate of single colonization $\gamma_{i}$ and clearance rate of co-colonization $\gamma_{i j}$ with $u_{11}=u_{12}=u_{21}=\nu_{1}$ and $u_{22}=\nu_{2}$. Figure 6 shows which combinations of $\Delta b$ and $\Delta \nu$, lead to one of the four scenarios: exclusion of strain 1 or 2 , coexistence or bistable state, for each $R_{0}$ and $k$, for assumed symmetry in $k_{i j}$ and in $p_{i j}^{i}=\frac{1}{2}$.

It can be seen that we can choose suitable values of relative fitness differences $\Delta b$ and $\Delta \nu$ to observe a given scenario, typically coexistence and bistability arise for relative advantage in one trait and relative disadvantage in the other, hence a trade-off between transmission and clearance. Notice that when $R_{0}$ or $k$ become larger, increasing relative coinfection prevalence in the corresponding neutral system, (i.e. reducing $\mu$ ), the possibility for coexistence or bistability expands in the system. Near the origin, it's harder to obtain coexistence or bistable state rather than competitive exclusion.

The results of Figure 6 can be used to connect our system's behavior to strain-specific basic reproduction numbers $R_{0}^{(1)}$ and $R_{0}^{(2)}$. In a model with coinfection, strain-specific $R_{0}$ are not sufficient to determine the long-time epidemiological competition between two strains. We show in Text S1 and in Figure S1 that even for the same values of $R_{0,1}$ and $R_{0,2}$ we can have different long-time scenarios between the two strains. The result in coinfection depends on the particular combination of traits, strain-specific transmission and clearance rates. In particular, even for the same strain-specific $R_{0}$ 's, a system with bigger variation in clearance rate between two strains is more sensitive to coinfection, and it is where coinfection parameters can shift the dynamics more easily away from competitive exclusion (Fig. S1 ). This is because of the advantage conferred to both strains by staying longer in mixed compartment $I_{12}$, where from they can have equal chance of transmission.

In Fig. 6 coinfection susceptibilities were assumed symmetric and coinfection clearance rate for mixed carriage was assumed biased toward the same clearance rate of single infection by strain 1: $u_{11}=u_{12}=u_{21}=$ $\nu_{1}$ and $u_{22}=\nu_{2}$. In another case (Figs. S3 34 , when we remove the bias in coinfection clearance assuming $u_{12}=u_{21}=0$, but allow variation in susceptibilities to coinfection, we see only three scenarios emerge, over all $\Delta b$ and $\Delta \nu$. In this case, depending on the structure of $\alpha_{i j}$, we observe either coexistence or bistability as a third possibility flanked by the two opposite exclusion steady states. 


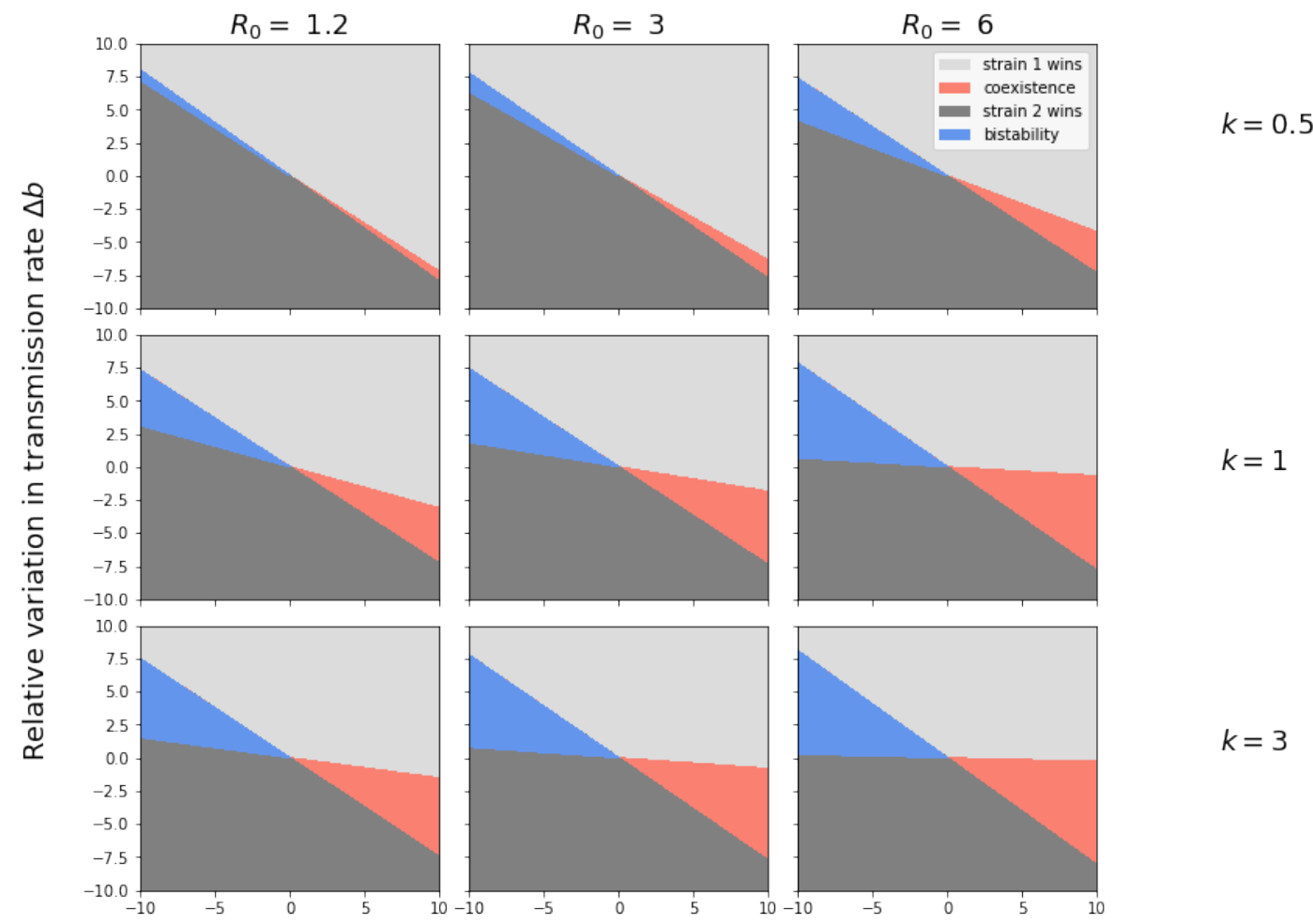

Relative variation in clearance rate $\Delta v$

Figure 6: Illustration of 4 possible outcomes, as a function of relative variation in transmission and clearance rate between two strains, for different values of $k$ and $R_{0}$. We highlight the respective regions in different colors, according to the critical relationship between $\Delta b, \Delta \nu, k$ and $R_{0}$ when perturbations happen only $\beta_{i}$, $\gamma_{i}$, and mixed coinfection clearance happens base on strain-specific clearance rates $\gamma_{i j}$ with $u_{11}=u_{12}=u_{21}=\nu_{1}$ and $u_{22}=\nu_{2}$. We choose the values $\gamma=1$ and $r=0.2$. Recall that the higher $\Delta \nu>0$, the higher the advantage of strain 1 in the system; and the higher $\Delta b>0$, the higher the advantage of strain 1 in the system. We observe coexistence and bistability arise only when the disadvantage in one trait is compensated by an advantage in the other. In particular coexistence is enabled when the disadvantaged strain 2 in clearance rate benefits from reduced clearance in mixed co-colonization with strain 1.

\section{How trait mean and variation impact coexistence frequencies}

Next, we zoom in from criteria for coexistence to the details of the coexistence equilibrium in a system with two strains that vary along multiple fitness dimensions. The general formula in terms of pairwise invasion fitnesses in Eqs. 3.1, when this equilibrium exists, is given by

$$
z_{1}^{*}=\frac{\lambda_{1}^{2}}{\lambda_{1}^{2}+\lambda_{2}^{1}} \quad z_{2} *=1-z_{1}^{*} .
$$

This allows explicit computation for any combination of strain-specific and strain-transcending parameters in the system. Below we focus on the case of variation in transmission rate $\beta_{i}$, clearance rate of infection $\gamma_{i}$ and in interaction coefficients via susceptibilities to coinfection $k_{i j}$. We assume the special case of $u_{i j}=0$ and initially no transmission biases in coinfection $p_{i j}^{i}=\frac{1}{2}$ (although we relax this later). We consider only coexistence regimes (Figure 7) and illustrate the equilibrium frequency of one of the strains (here $z_{2}^{*}$ ), as a function of trait mean and variation between strains. Using the explicit formula $z_{2}^{*}=\lambda_{2}^{1} /\left(\lambda_{1}^{2}+\lambda_{2}^{1}\right)$, we explore this quantity numerically for two values of co-colonization interaction mean coefficient: $k=1.5$ and $k=0.2$, and symmetric cross-strain interactions $\alpha_{12}=\alpha_{21}>\alpha_{11}=\alpha_{22}$. We consider $\left(\alpha_{i j}\right)_{i j}=\left(\begin{array}{cc}0 & \sqrt{2} \\ \sqrt{2} & 0\end{array}\right)$ but the general formula for other cases is straightforward (see Text S3). The criteria enabling coexistence are derived below. 

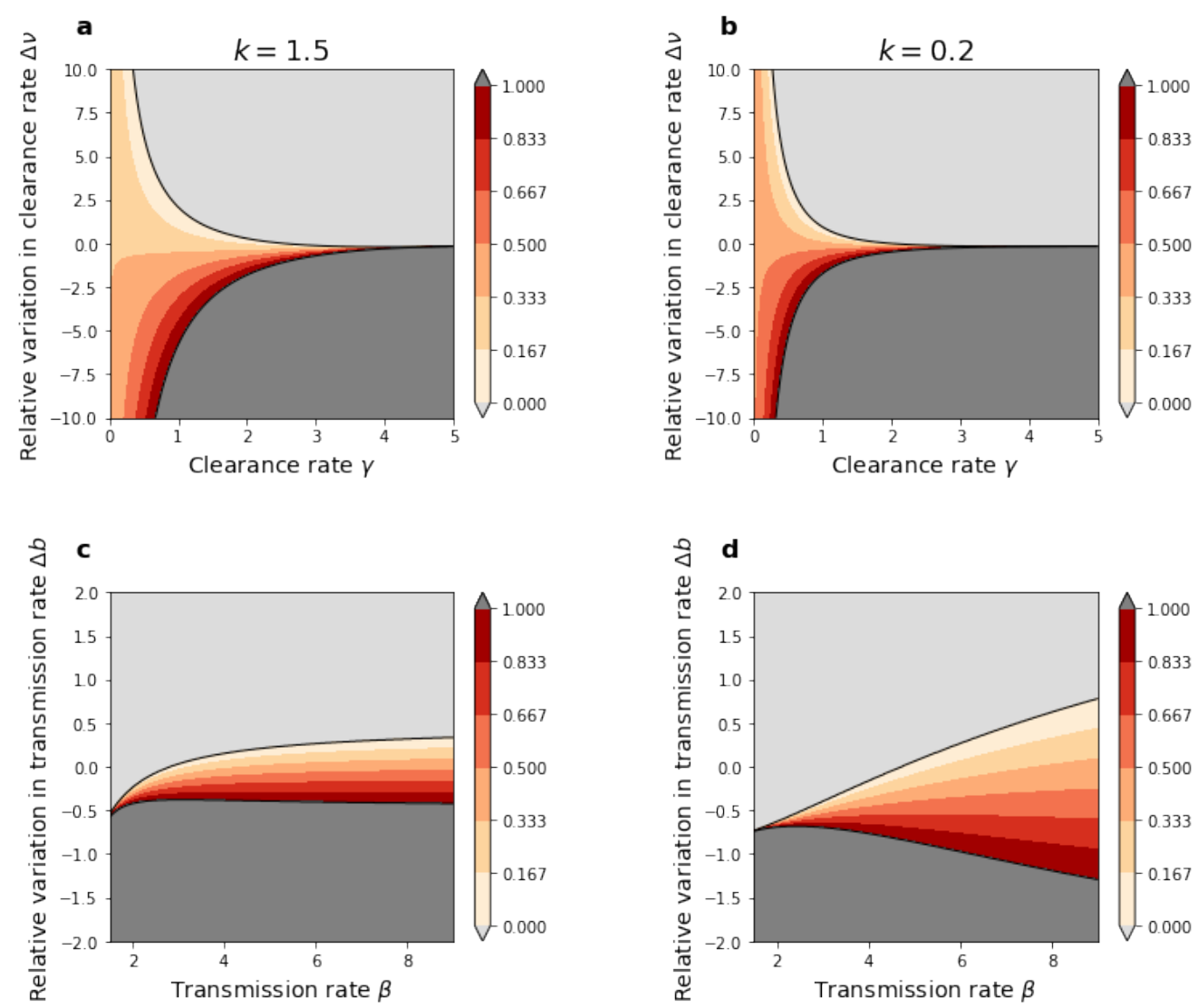

Figure 7: Strain coexistence frequencies depend on a critical interplay between trait mean and variation. We plot the coexistence equilibrium frequency of strain 2 (intermediate shading), as a function of $(\beta, \Delta b)$ and $(\gamma, \Delta \nu)$ for higher coinfection prevalence (left) and lower coinfection prevalence (right). a-b. Effect of clearance rate. In (a-b) we assume $\beta=5.3$ and $\Delta b=0.15$. In these figures, we vary $\gamma$ in $0 \leq \gamma \leq 5$ and $\beta=5.3$, to make $R_{0}>1$. c-d. Effect of transmission rate. In (c-d) we assume $\gamma=1$ and $\Delta \nu=1$ as fixed. We vary $\beta$ between 1.5 and 9 , ensuring $R_{0}>1$. Right-column subplots reflect a system with more competition in co-colonization, hence lower coinfection prevalence $\left(\mu\right.$ higher) than the left-column subplots. The light grey region represents the exclusion of strain $2-z_{2}^{*}=0$ and the dark grey region is for exclusion of strain 1 from the system i.e. $z_{2}^{*}=1$. We choose $r=0.3$ and the matrix $\left(\alpha_{i j}\right)$ to be $\left(\begin{array}{cc}0 & \sqrt{2} \\ \sqrt{2} & 0\end{array}\right)$ thus unbiased in terms of favouring either strain. The co-colonization clearance rate is also assumed unbiased, and equal to the mean. The black lines denote the border lines for which coexistence is no longer possible and the system shifts to either exclusion of strain 1 (yellow) or exclusion of strain 2 (blue). In a-b, the lines are denoted by $T_{1}(\gamma)$ and $T_{2}(\gamma)$, and given by 5.2 and 5.3 . In c-d, the graphs of $S_{1}(\beta)$ and $S_{2}(\beta)$ are hyperbolic, given by explicit equations $[5.5)$ and $(5.6)$.

\subsection{Mean and variation in clearance rate of single infection}

By 4.3 , the equation of boundary $z_{2}^{*}=0$ is equivalent to $\lambda_{2}^{1}=0$ which can be written explicitly as:

$$
\frac{\gamma}{2(\gamma+r)} \frac{1}{1+\frac{1}{\mu}}\left(1+\frac{1}{1+\frac{1}{\mu}}\right) \Delta \nu=-\Delta b-\frac{\sqrt{2}}{2}\left(R_{0}-1\right) \frac{\mu}{(\mu+1)^{2}}
$$

which, by substituting $\frac{1}{\mu}=k\left(\frac{\beta}{\gamma+r}-1\right)$, becomes:

$$
\Delta \nu=T_{1}(\gamma), \quad T_{1}(\gamma)=-\Delta b \frac{2[\gamma+r+k(\beta-\gamma-r)]^{2}}{\gamma(2 \gamma+2 r+k(\beta-\gamma-r))}-\sqrt{2} \frac{k}{\gamma} \frac{(\beta-\gamma-r)^{2}}{2(\gamma+r)+k(\beta-\gamma-r)}
$$

Analogously, we can compute the equation of boundary $z_{2}^{*}=1$, thus $\lambda_{1}^{2}=0$, which is

$$
\Delta \nu=T_{2}(\gamma), \quad T_{2}(\gamma)=-\Delta b \frac{2[\gamma+r+k(\beta-\gamma-r)]^{2}}{\gamma(2 \gamma+2 r+k(\beta-\gamma-r))}+\sqrt{2} \frac{k}{\gamma} \frac{(\beta-\gamma-r)^{2}}{2(\gamma+r)+k(\beta-\gamma-r)}
$$


Combining (5.2) and (5.3) we have the condition for 2-strain coexistence between these boundaries, expressed as an inequality for the variation $\Delta \nu$ dependent on the mean $\gamma$ :

$$
\text { Stable Coexistence } \Longleftrightarrow T_{1}(\gamma) \leq \Delta \nu \leq T_{2}(\gamma) \text {. }
$$

The figures $7 \mathrm{~b}$ and $7 \mathrm{~b}$ show critical interplay between mean and variation clearance rates of infection $\gamma_{i}$. The light color region in this figure is the region displays coexistence phenomenon, corresponding to the space between two curves which present equations (5.2) and (5.3). The contour shading corresponds to the relative frequency of strain 2. In these figures, we can observe that by decreasing co-colonization efficiency $k$, we can decrease the possibility of coexistence at the same variation in clearance rate $\Delta \nu$ (7b compared to $7 \mathrm{~b})$. For $\gamma$ small enough, which increases overall prevalence in the system, less values of $\Delta \nu$ lead to coexistence. When $\Delta \nu$ becomes larger, the relative frequency of strain 2 , decreases.

\subsection{Mean and variation in transmission rate}

Similarly to (5.2) and (5.3) for mean and variation in clearance rate (duration of carriage), and the same assumptions about the other parameters, we can find the equation of boundary $z_{2}^{*}=0$ as a function of $(\beta, \triangle b)$ as follows:

$$
\Delta b=S_{2}(\beta), \quad S_{2}(\beta)=-\Delta \nu \frac{\gamma(2 \gamma+2 r+k(\beta-\gamma-r))}{2[\gamma+r+k(\beta-\gamma-r)]^{2}}+\frac{\sqrt{2}}{2} \frac{k(\beta-\gamma-r)^{2}}{[\gamma+r+k(\beta-\gamma-r)]^{2}}
$$

The equation of the boundary $z_{2}^{*}=1$ as a function of $(\beta, \triangle b)$ is:

$$
\Delta b=S_{1}(\beta), \quad S_{1}(\beta)=-\Delta \nu \frac{\gamma(2 \gamma+2 r+k(\beta-\gamma-r))}{2[\gamma+r+k(\beta-\gamma-r)]^{2}}-\frac{\sqrt{2}}{2} \frac{k(\beta-\gamma-r)^{2}}{[\gamma+r+k(\beta-\gamma-r)]^{2}} .
$$

Combining (5.5) and (5.6), we have the condition on intermediate values of $\Delta b$ that allow 2-strain coexistence:

$$
\text { Stable Coexistence } \Longleftrightarrow S_{1}(\beta) \leq \Delta b \leq S_{2}(\beta) \text {. }
$$

Two figures $7 \mathrm{f}$ and $7 \mathrm{~d}$ show the value of strain 2 in case of coexistence as a function of mean and variation in transmission rates $\Delta b$. In contrast to $7 \mathrm{k}$ and $7 \mathrm{~b}$, by decreasing co-colonization vulnerability $k$, we increase the possibility of coexistence at the same variation in transmissibility between strains $\Delta b$. This also means that for larger $\beta$, more values of $\Delta b$ lead to coexistence. When $\Delta b$ becomes larger, increasing the transmission advantage of strain 1 , the equilibrium frequency of strain 2 , decreases. This is similar to Figure $7 \mathrm{a}$ and $7 \mathrm{~b}$.

\subsection{Coexistence strain frequencies are explicit}

While the entire frequency dynamics for each strain and for all time $\left(z_{i}(\tau)\right)$ is fully explicit in the system, via the replicator equation, so is also the final equilibrium. The value of the equilibrium frequency of strain $2, z_{2}^{*}$ under the coexistence regimes studied above is given by:

$$
z_{2}^{*}=\frac{1}{2}-\gamma \frac{2(\gamma+r)+k(\beta-\gamma-r)}{2 \sqrt{2} k(\beta-\gamma-r)^{2}} \Delta \nu-\frac{[\gamma+r+k(\beta-\gamma-r)]^{2}}{\sqrt{2} k(\beta-\gamma-r)^{2}} \Delta b,
$$

and can be seen to be an explicit function of mean parameters (e.g. $\beta, \gamma$ ) as well as variation between strains $(\Delta b, \Delta \nu)$. Here, because coinfection interactions are assumed symmetric, it becomes obvious that any differences between strains will make the frequency deviate from the expected frequency of $1 / 2$ under balancing selection. If $\Delta \nu, \Delta b>0$, then strain 1 has an absolute advantage and $z_{2}$ will always be inferior than $1 / 2$, if it coexists. However, if the advantage is only in one trait and not in the other $(\Delta \nu \Delta b$ of different sign), then strain 2 can increase its equilibrium frequency above $1 / 2$.

More generally, for the rescaled co-colonization susceptibilities matrix being symmetric and satisfying: $\alpha_{i i}=\alpha_{j j}=\alpha_{11}$ and $\alpha_{i j}=\alpha_{j i}=\alpha_{12}$, we can write the expression for strain 2 equilibrium frequency, more compactly as a function of $\mu, k$ and $R_{0}$ constituent parameters, mean transmission rate $\beta$, clearance rate $\gamma$ and average host lifespan $1 / r$ :

$$
z_{2}^{*}=\frac{1}{2}\left[1-\frac{k}{\alpha_{12}-\alpha_{11}}\left(\mu(2 \mu+1) \frac{\gamma}{\gamma+r} \Delta \nu+2(\mu+1)^{2} \Delta b\right)\right]
$$

One can notice that variation in each trait $\Delta b$ and $\Delta \nu$ have their own distinct nonlinear scaling factors for how they impact on ultimate strain success at the epidemiological level, depending directly on the prevalence of 
co-colonization in the system via the parameter $\mu=I^{*} / D^{*}$. We can immediately see from this formula, how the predicted coexistence level among two strains is attributable to clear mechanisms and clearly identifiable biological differences between strains, which are explicitly weighted by epidemiologic constants.

Even more generally, if in addition there are biases in strain transmission probabilities from co-colonized hosts carrying a mixture of strains, recalling that $\Delta \omega=\omega_{12}^{1}-\omega_{21}^{2}$ the formula (5.9) now reads

$$
z_{2}^{*}=\frac{1}{2}\left[1-\frac{k}{\alpha_{12}-\alpha_{11}}\left(\mu(2 \mu+1) \frac{\gamma}{\gamma+r} \Delta \nu+2(\mu+1)^{2} \Delta b+2(\mu+1) \Delta \omega\right)\right]
$$

where we can straightforwardly see the contribution of the precedence effect in transmission from co-colonization as a force in coexistence hierarchies between two strains. Indeed, for any value of coinfection prevalence and overall $R_{0}$, hence for any $\mu$, the relative contribution of the transmission rate differential between strains $(\Delta b)$ is higher than that of the transmission bias differential from coinfected hosts $(\Delta \omega)$. It is also interesting to notice in the above expression for $z_{2}^{*}$ that the relative contribution of the between-strain difference in duration of carriage $(\Delta \nu)$ depends on $\mu$ but also on the absolute value of the duration of colonization itself $\gamma$. Essentially, keeping all else fixed, differences in duration of carriage between two strains matter more for their relative fitness, if the colonization episodes are shorter ( $\gamma$ higher), and if hosts are longer lived ( $r$ higher).

Perfectly balancing selection, in this model, under a given $k \neq 0$, would require that the linear combination of $\Delta \nu, \Delta b, \Delta \omega$ in the round brackets be equal to zero. Trait variation possibilities satisfying this requirement are infinite and constitute a plane in 3-d in this case, necessarily encoding trade-offs across different fitness dimensions that would as a whole lead to the same epidemiologic fitness for the two strains. Other particular values of $z_{2}^{*}$ can be studied explicitly and investigated similarly in terms of the constraints implied for the linear combination of $\Delta \nu, \Delta b, \Delta \omega$, and other fitness dimensions eventually, if such variation exists.

\section{Model applications: a roadmap}

In earlier theoretical work, interaction among co-infecting agents has been assumed to occur only between different strains, and studied for arbitrary infection multiplicity Adler and Brunet (1991). Later evolutionary frameworks, based on (van Baalen and Sabelis, 1995), have considered a full model including same-strain coinfection, but modeling vulnerability to co-infection with a single parameter (Alizon, 2013). This aggregation of within- and between- strain interactions into a net parameter can be found in other co-infection models, considering altered susceptibility to coinfection in the context of disease persistence (Gaivão et al., 2017), and diversity in other traits, e.g. virulence (Alizon et al., 2013) and antibiotic resistance (Hansen and Day, 2014). These studies highlight the importance of coinfection and its epidemiological details for persistence and evolution of microorganisms. Sometimes very complex multi-scale models have been invoked to generate coexistence between strains via coinfection, embedding an explicit within-host dynamics framework (Davies et al. 2019). We argue that many such coinfection models could be mapped to phenomena in the overarching model proposed here, as special cases, or expansions of a particular parameter.

With the here-proposed explicit framework, the impact of coinfection becomes very easy to understand, via the role of the parameter $\mu$, given by the ratio of single - to co-infection prevalence in the system, which modulates the relative weight of different trait asymmetries $\left(\theta_{i}\right.$ 's) among strains, and even tuning the net asymmetries in some traits, as is the case for $k_{i j}$. This role of coinfection (in terms of 2 global determining parameters $R_{0}, k$ ) can be studied at a deeper level, at a higher resolution in terms of potential asymmetries within and between strains, and in an entirely analytically-explicit manner which enables precise predictions. These advantages can lead to new applications to study coexistence and vaccination effects in polymorphic systems, going beyond current theoretical insights (Lipsitch, 1997: Gjini et al., 2016). Similarly, our modeling framework could also help obtain clear and direct analytical insights into antibiotic resistance evolution, as an alternative or as a complement to the more cumbersome simulation route (Davies et al., 2019). Below we sketch briefly some ways in which the model can be applied.

\subsection{Antibiotic treatment, fitness costs and competitive release}

We can apply this model to understand epidemiological competition dynamics between two strains under antibiotics, co-circulating in a host population with the possibility of coinfection. In its simplest form, broadspectrum antibiotic treatment can be modelled as an increase in global clearance rate of colonization $\gamma$, keeping fixed all other parameters between strains. Suppose strain 1 is superior in transmission, with strain 2 suffering a relative fitness cost $\Delta b>0$. For variation in duration of carriage we explore both an advantage to strain $1 \Delta \nu>0$ or advantage to strain $2 \Delta \nu<0$. Without perturbation in coinfection parameters, under such scenario, there would be competitive exclusion of the less fit strain, seen earlier in Section 3.1. 
But under interactions through coinfection vulnerabilities, coexistence among the two strains is possible. Thus we may explore, the frequency of strain 2, under such scenario, which would then correspond to the variable $z_{2}$ in our model. We examine how the equilibrium value of the frequency of strain 2 , varies as a function of $\gamma$ (or total broad-spectrum antibiotic treatment), for different values of strain variation in relative duration of carriage (clearance rate) $\Delta \nu$, and relative transmissibility $\Delta b$.

Fixing for example the rescaled co-infection susceptibility matrix to $\alpha_{i i}=\alpha_{j j}=0$ and $\alpha_{i j}=\alpha_{j i}=\sqrt{2}$, corresponds to the case analyzed earlier, where within-strain susceptibilities to coinfection are lower than between-strain susceptibilities, a condition that a-priori favours coexistence. Hence, applying the earlier results, we have that for any $(\beta, \gamma, \Delta b, \Delta \nu)$, stable coexistence of two strains is possible only for $T_{1}(\gamma) \leq$ $\Delta \nu \leq T_{2}(\gamma)$. The value of $z_{2}^{*}$ under such condition is given by Eq. 5.8. Thus, in Figure 8, we only consider the values of global clearance rate $\gamma$ guaranteeing coexistence. It is interesting to point out that depending on how the fitness differential between the strain 1 and its competitor strain 2 , is manifested $(\Delta b, \Delta \nu)$, increasing antibiotic administration in a population can have opposing effects: it can increase or decrease the prevalence of a focal strain (see Fig 8 a solid vs. dashed lines). This behavior can be understood in full analytic detail because of the explicit expression for strain frequencies, allowing us to compute and verify directly the first and second derivatives of $z_{2}$ with respect to $\gamma$ (see Supplementary Material S3).
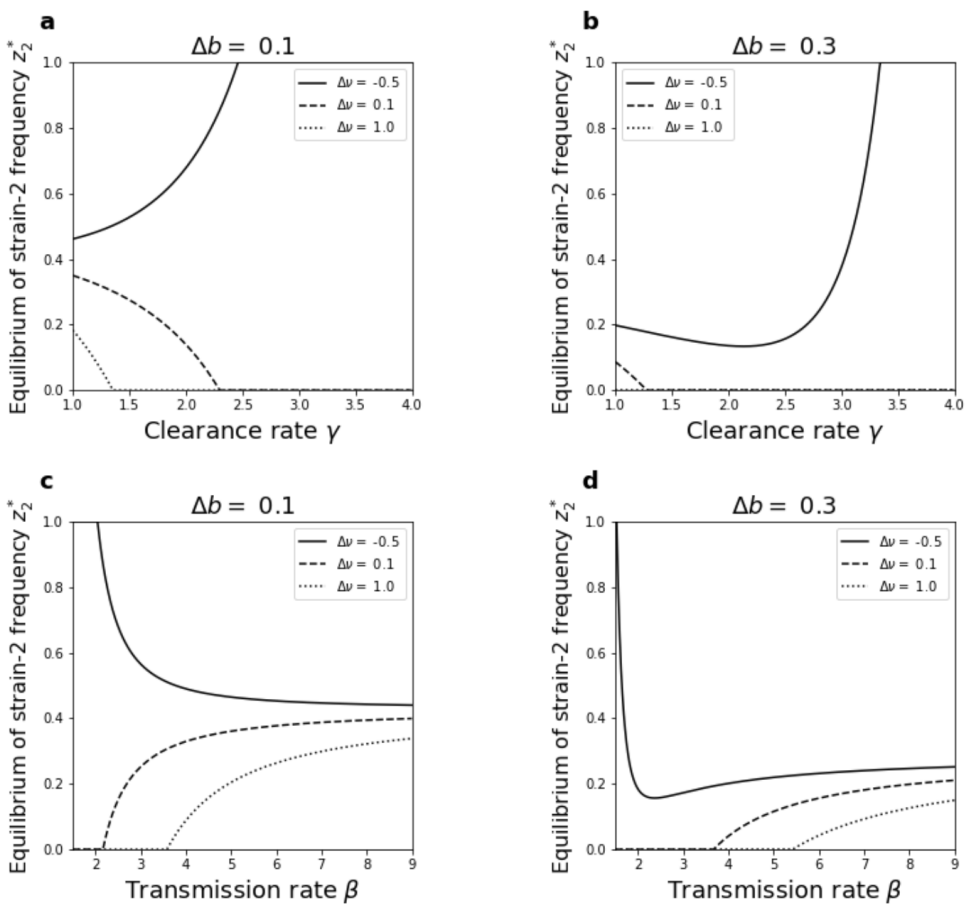

\section{Intervention}

Figure 8: Increasing global clearance or global transmission can have opposite effects on single strain frequency at the endemic equilibrium, depending on underlying trait variation. We plot the frequency of strain $2, z_{2}^{*}$ at the coexistence equilibrium (Eq. 5.8), as a function of mean transmission rate and mean clearance rate. Variation among 2-strains is encoded in the transmission and clearance rate axes: $\beta_{i}$ and $\gamma_{i}$, and co-colonization vulnerabilities $k_{i j}$. In this simulation, we choose $r=0.2, k=1$ and the matrix of standardized interactions is assumed symmetric $\left(\alpha_{i j}\right)=\left(\begin{array}{cc}0 & \sqrt{2} \\ \sqrt{2} & 0\end{array}\right)$, favouring coexistence with $\alpha_{i j}=\alpha_{j i}>\alpha_{i i}=\alpha_{j j}$. a-b. Equilibrium frequency as a function of strain-transcending $\gamma$. We plot $z_{2}^{*}$ as a function of mean clearance rate $\gamma$ (varied between 1 and 4 ) for 2 cases of fitness differentials in transmission $\Delta b$ and 3 cases of variability in clearance $\Delta \nu$. The global transmission rate is $\beta=4.5$ to ensure $R_{0} \geq 1$. c-d. Equilibrium frequency as a function of strain-transcending $\beta$. We plot $z_{2}^{*}$, as a function of mean transmission rate $\beta$ (varied between 1.5 and 9) for 2 cases of different variation $\Delta b$ and 3 cases of $\Delta \nu$. In these plots, overall clearance rate is held fixed at $\gamma=1$ to ensure $R_{0} \geq 1$.

To understand the additional effects of possible variations in transmission probability from mixed coinfection between strains, we have repeated the same simulations with $\Delta w<0$, favouring strain 2 (Supplementary figure S7). It is clear that also this dimension of fitness (within-host advantage) has a substantial effect on the net competitive dynamics between the two strains, and in particular, in this case, enhances the possibility of two-strain coexistence. 


\subsection{Explicit link for vaccination, coexistence and strain replacement}

Similarly, universal vaccination that protects against both strains could be modelled, to a first-order approximation, as a global reduction of $\beta$ in the system, realized via reduced average susceptibility of all hosts to infection. In figures 8 and $8 \mathrm{~d}$, we explore the effect of a universal reduction in $\beta$ on the relative prevalence of two strains. As shown earlier, coexistence is possible only for $S_{1}(\beta) \leq \Delta b \leq S_{2}(\beta)$. The value of $z_{2}^{*}$ under such condition is given by Equation 5.8. Plotting this as a function of $\beta$ in Figures 8 c and 8 d, we observe again that changes in strain-transcending transmissibility can have opposing effects on prevalence of a focal strain (here strain 2). They can either favour its increase in prevalence or its decrease, depending on the underlying basic trait variation $(\Delta b, \Delta \nu)$, as well as on coinfection parameters ( $k_{i j}$ or others).

Turning the inequalities around, another way to interpret the critical borders for $\Delta b$ is with regards to a strain-specific vaccine. For example, assuming universal coverage, in order to predict the minimal vaccine efficacy needed to exclude a given strain (or group of strains) from the system, given everything else fixed at pre-vaccine baseline, we can use the model to extract the $\Delta b$ that violates the coexistence inequality criterion: $S_{1}(\beta) \leq \Delta b \leq S_{2}(\beta)$. Notice that this criterion specifically implies that in populations with different overall $\beta$ a different $\Delta b$ (targeted vaccine efficacy) may be needed to reach exclusion. In the case of $\Delta b<S_{1}(\beta)$ we would ensure exclusion of strain 1 from the system, whereas making $\Delta b>S_{2}(\beta)$ would shift the system to the exclusion of strain 2 steady state.

\subsection{Effects of host demography on 2-strain competition}

Changes in natural susceptible recruitment rate (equal host mortality rate) $r$ affect $R_{0}$ in the system, which also determines $\mu$, besides appearing itself also explicitly in Eq 5.9 . So even with everything else fixed $(\beta, \gamma, k$ and $\Delta b, \Delta \nu)$ it is possible to influence competitive dynamics between strain 1 and strain 2 , just via host demography. Increasing host mortality rate, decreases $R_{0}$ in the system which increases $\mu$ and hence gives a larger weight to the trait variation in clearance rate of infection $\Delta \nu$. Increasing host turnover rate might then enable coexistence of two strains (maintenance of the less fit strain e.g. strain 2) because it could amplify, and even overturn, the relative advantage in duration of carriage $(\Delta \nu<0)$ versus the disadvantage in transmissibility $(\Delta b>0)$. This would imply that in different populations, with different rates of susceptible host turnover, the dynamics of the same two strains could be different. Thanks to this model, all these mechanisms and special cases in competitive dynamics between two closely-related strains at the epidemiological scale can be studied in a fully parameter-explicit and analytical manner, which should promote easier and more direct testable links with data.

\subsection{Dynamical transitions: from $N=2$ to the $N$-strain network}

Throughout this study, we have shown explicitly and illustrated in detail how global parameters of the neutral model, embedded in the center of the dynamics, can shift qualitatively and quantitatively the net competitive outcome for any given pair of strains. Recall that the $N=2$ system forms the basic unit in the full competitive network among an arbitrary number $N$ of strains, and that qualitative shifts in each network 'edge', as a function of global parameters, can have far-reaching effects on the collective dynamics among multiple strains, even when strains differ just in co-colonization interactions (Gjini and Madec, 2021a). Having exposed further nonlinear gradient effects of $\mu, R_{0}, k, \gamma, r$ in the more complete 2-strain system with variation along 5 fitness dimensions, opens the way towards deeper analysis of their higher-level effects on the $N-$ strain dynamics and coexistence (Le et al., 2021). Studying dynamical transitions mediated via coinfection prevalence and strain-transcending epidemiological parameters in the full system is the natural and exciting next step.

\section{Discussion}

Coinfection is an important aspect of many infectious diseases. There are substantial modeling efforts dedicated to co-infection in the last decades (Adler and Brunet, 1991, Lipsitch, 1997, Gjini et al., 2016, Alizon et al., 2013, Hansen and Day, 2014; Davies et al., 2019). Yet, simple and sufficiently general mathematical frameworks to analyze and unify the full spectrum of hierarchical patterns emerging from co-colonization (co-infection) interactions and variation in other fitness dimensions between two strains are missing. Here, we contribute to fill this gap, thanks to a model reduction obtained after assuming strain similarity (Le et al. 2021). Focusing on $N=2$, here we have modeled simultaneously 5 fitness dimensions where two strains can differ, and used the decomoposition into two timescales to simplify their dynamics: neutral dynamics between types on a fast timescale and non-neutral selective processes on a slow timescale, driven explicitly by trait variation, going beyond (Gjini and Madec, 2017) where only pairwise co-colonization coefficients (a single 'trait') were studied. 
Many studies of coinfection are either totally epidemiological in nature (Martcheva, 2009, Thieme, 2007, Lipsitch, 1997), exploring transmission dynamics of infectious agents in a host population, or they focus on evolution of specific pathogen traits (often virulence) (Mosquera and Adler, 1998, Alizon et al., 2013) using the coinfection framework developed by (van Baalen and Sabelis, 1995) for microparasites causing persistent infections. The latter group of studies, typically derive the conditions of invasion of a rare mutant in a host population already infected by a resident strain, following adaptive dynamics theory (Meszéna et al., 2005), where it is further assumed that the resident strain is at equilibrium, that is, that the densities of susceptible, singly-infected and coinfected hosts have reached their equilibrium values. Invader fitness is then evaluated using the basic reproduction ratio (van Baalen and Sabelis, 1995), where it becomes clear that the fitness of a mutant strain is the sum of two components: the fitness achieved through the infection of susceptible hosts and the fitness achieved through the infection of hosts already infected by the resident. Typically by analyzing whether the reproduction ratio is greater than or lower than 1, conditions for successful or unsuccessful invasion, and ultimate evolutionary dynamics for the trait in consideration are established. However, sometimes the criteria derived in such models can be model-dependent, involve cumbersome mathematical expressions, and may not provide immediate comprehensive insight into the biological mechanisms.

In the present work, with a rather generic model, we have revealed coexistence and competition mechanisms in their bare essence, and have integrated, generalized and advanced analytically these two perspectives on coinfection. We have linked population dynamics of endemic transmission with slow selective dynamics in strain trait space. We have generalized single trait evolution to multiple trait evolution, exploring fitness variation along 5 dimensions between two strains: transmission, clearance, vulnerability to coinfection, duration of coinfection, and transmission biases from mixed coinfection. We have also presented an analytical expression for explicit frequency dynamics between two strains, under an endemic global equilibrium, which allows to use the well-known replicator equation to make predictions for relative strain abundances over time, and highlights how the means of different traits directly affect their joint variation dynamics. We have illustrated only special cases, where the traits are uncorrelated. Possible co-variation constraints or trade-offs between different traits (e.g. transmission-clearance, or transmission -competition in co-colonization) can be studied under the same analytically-explicit framework, provided they do not violate the similarity assumption.

We believe this analytical approach, derived for general $N$ in (Le et al., 2021), starting with a complete coinfection structure (involving both same strain and mixed co-colonization as in van Baalen and Sabelis 1995, Alizon, 2013, Gjini et al., 2016)), and made possible via timescale separation and model reduction under the assumption of strain similarity (following (Gjini and Madec, 2017; Madec and Gjini, 2020)), can have countless further theoretical and practical applications. Understanding more deeply multiple-trait selection in coinfection systems will enable easier and more direct insights on several important health challenges, including antibiotic resistance, virulence evolution, optimal immunization and patterns of diversity in multistrain endemic pathogens.

\section{Acknowledgements}

Thi Minh Thao Le was supported with a Ph.D. scholarship by the University of Tours. Erida Gjini was supported by PESSOA grant 5666/44637YE awarded by Portuguese Foundation for Science and Technology (FCT), and cooperative agreement 2020-2001-230-Y20V7 as Le Studium Visiting Researcher, from Loire Valley Institute of Advanced Studies, Orleans and Tours, France, hosted by Institut Denis Poisson (CNRS/Universite de Tours/Universite d'Orleans) in 2021.

\section{References}

Abdullahi, O., A. Karani, C. C. Tigoi, D. Mugo, S. Kungu, E. Wanjiru, J. Jomo, R. Musyimi, M. Lipsitch, and J. A. G. Scott (2012). Rates of acquisition and clearance of pneumococcal serotypes in the nasopharynges of children in kilifi district, kenya. Journal of Infectious Diseases 206(7), 1020-1029.

Adler, F. R. and R. C. Brunet (1991). The dynamics of simultaneous infections with altered susceptibilities. Theoretical population biology 40(3), 369-410.

Alizon, S. (2008). Decreased overall virulence in coinfected hosts leads to the persistence of virulent parasites. The American Naturalist 172(2), E67-E79.

Alizon, S. (2013). Co-infection and super-infection models in evolutionary epidemiology. Interface focus 3(6), 20130031.

Alizon, S., J. C. de Roode, and Y. Michalakis (2013). Multiple infections and the evolution of virulence. Ecology letters 16(4), 556-567. 
Chen, L., F. Ghanbarnejad, and D. Brockmann (2017). Fundamental properties of cooperative contagion processes. New J. of Phys. 19(10).

Cobey, S. and M. Lipsitch (2013). Pathogen diversity and hidden regimes of apparent competition. The American Naturalist 181(1), 12-24.

Cohen, T., P. D. van Helden, D. Wilson, C. Colijn, M. M. McLaughlin, I. Abubakar, and R. M. Warren (2012). Mixed-strain mycobacterium tuberculosis infections and the implications for tuberculosis treatment and control. Clinical microbiology reviews 25(4), 708-719.

Davies, N. G., S. Flasche, M. Jit, and K. E. Atkins (2019). Within-host dynamics shape antibiotic resistance in commensal bacteria. Nature ecology $\&$ evolution 3(3), 440.

De Roode, J. C., M. E. Helinski, M. A. Anwar, and A. F. Read (2005). Dynamics of multiple infection and within-host competition in genetically diverse malaria infections. The American Naturalist 166 (5), 531-542.

Furuya-Kanamori, L., S. Liang, G. Milinovich, R. J. S. Magalhaes, A. C. Clements, W. Hu, P. Brasil, F. D. Frentiu, R. Dunning, and L. Yakob (2016). Co-distribution and co-infection of chikungunya and dengue viruses. BMC infectious diseases 16(1), 1-11.

Gaivão, M., F. Dionisio, and E. Gjini (2017). Transmission fitness in co-colonization and the persistence of bacterial pathogens. Bulletin of mathematical biology 79(9), 2068-2087.

Geritz, S. A., G. Mesze, J. A. Metz, et al. (1998). Evolutionarily singular strategies and the adaptive growth and branching of the evolutionary tree. Evolutionary ecology 12(1), 35-57.

Gjini, E. and S. Madec (2017). A slow-fast dynamic decomposition links neutral and non-neutral coexistence in interacting multi-strain pathogens. Theoretical Ecology 10(1), 129-141.

Gjini, E. and S. Madec (2021a). The ratio of single to co-colonization is key to complexity in interacting systems with multiple strains. Ecology and Evolution (in press) doi:10.1002/ece3.7259.

Gjini, E. and S. Madec (2021b). Towards a mathematical understanding of colonization resistance. bioRxiv.

Gjini, E., C. Valente, R. Sá-Leão, and M. G. M. Gomes (2016). How direct competition shapes coexistence and vaccine effects in multi-strain pathogen systems. Journal of Theoretical Biology 388, 50-60.

Halliday, F. W., R. M. Penczykowski, B. Barrès, J. L. Eck, E. Numminen, and A.-L. Laine (2020). Facilitative priority effects drive parasite assembly under coinfection. Nature Ecology \& Evolution 4(11), 1510-1521.

Hansen, J. and T. Day (2014). Coinfection and the evolution of drug resistance. Journal of evolutionary biology 27(12), 2595-2604.

Hofbauer, J. and K. Sigmund (2003). Evolutionary game dynamics. Bulletin of the American Mathematical Society 40(4), 479-519.

Le, T. M. T., E. Gjini, and S. Madec (2021). Quasi-neutral dynamics in a coinfection system with n strains and multiple trait variation. aRxiv.

Levin, S. and D. Pimentel (1981). Selection of intermediate rates of increase in parasite-host systems. The American Naturalist 117(3), 308-315.

Lipsitch, M. (1997). Vaccination against colonizing bacteria with multiple serotypes. PNAS 94(12), 65716576 .

Lipsitch, M., C. Colijn, T. Cohen, W. P. Hanage, and C. Fraser (2009). No coexistence for free: neutral null models for multistrain pathogens. Epidemics 1(1), 2-13.

Madec, S. and E. Gjini (2020). Predicting n-strain coexistence from co-colonization interactions: epidemiology meets ecology and the replicator equation. Bulletin of mathematical biology 82(11), 1-26.

Margolis, E., A. Yates, and B. R. Levin (2010). The ecology of nasal colonization of Streptococcus pneumoniae, Haemophilus influenzae and Staphylococcus aureus: the role of competition and interactions with host's immune response. BMC Microbiology 10(1), 59.

Martcheva, M. (2009). A non-autonomous multi-strain sis epidemic model. Journal of biological dynamics 3(23), 235-251. 
May, R. M. and M. A. Nowak (1995). Coinfection and the evolution of parasite virulence. Proceedings of the Royal Society of London. Series B: Biological Sciences 261(1361), 209-215.

Meszéna, G., M. Gyllenberg, F. J. Jacobs, and J. A. Metz (2005). Link between population dynamics and dynamics of darwinian evolution. Physical review letters 95(7), 078105.

Mosquera, J. and F. R. Adler (1998). Evolution of virulence: a unified framework for coinfection and superinfection. Journal of Theoretical Biology 195(3), 293-313.

Nicoli, E. J., D. Ayabina, C. L. Trotter, K. M. Turner, and C. Colijn (2015). Competition, coinfection and strain replacement in models of bordetella pertussis. Theoretical population biology 103, 84-92.

Nowak, M. A. and R. M. May (1994). Superinfection and the evolution of parasite virulence. Proceedings of the Royal Society of London. Series B: Biological Sciences 255(1342), 81-89.

Pinotti, F., F. Ghanbarnejad, P. Hövel, and C. Poletto (2019). Interplay between competitive and cooperative interactions in a three-player pathogen system. arXiv preprint arXiv:1912.07289.

Susi, H., B. Barrès, P. F. Vale, and A.-L. Laine (2015). Co-infection alters population dynamics of infectious disease. Nature communications $6(1), 1-8$.

Thieme, H. R. (2007). Pathogen competition and coexistence and the evolution of virulence. In Mathematics for Life Science and Medicine, pp. 123-153. Springer.

van Baalen, M. and M. W. Sabelis (1995). The dynamics of multiple infection and the evolution of virulence. American Naturalist, 881-910.

Zhang, Y., K. Auranen, and M. Eichner (2004). The influence of competition and vaccination on the coexistence of two pneumococcal serotypes. Epidemiology and Infection 132(06), 1073-1081. 


\section{Supplementary information}

Disentangling how multiple traits drive 2 strain frequencies in SIS dynamics with coinfection Thi Minh Thao Le ${ }^{1, *}$, Sten Madec ${ }^{1,+}$, Erida Gjini ${ }^{2, \dagger}$

${ }^{1}$ Laboratory of Mathematics, University of Tours, Tours, France,

${ }^{2}$ Center for Stochastic and Computational Mathematics, Instituto Superior Tecnico, Lisbon, Portugal

*Thi-minh-thao.Le@lmpt.univ-tours.fr, ${ }^{+}$Sten.Madec@lmpt.univ-tours.fr, ${ }^{\dagger}$ erida.gjini@tecnico.ulisboa.pt

\section{S1 Strain-specific $R_{0}$ in co-infection vs. actual trait variation}

We may consider the relations between the basic reproductive numbers $R_{0,1}$ and $R_{0,2}$ in determining the winner in the case of exclusion. We recall the basic reproductive number of each strain, see Le et al. (2021) as follows

$$
R_{0,1}=\frac{\beta}{m}+\epsilon \frac{\beta}{m}\left(\frac{b_{1}}{\beta}-\frac{\nu_{1}}{m}\right)+O\left(\epsilon^{2}\right), \quad R_{0,2}=\frac{\beta}{m}+\epsilon \frac{\beta}{m}\left(\frac{b_{2}}{\beta}-\frac{\nu_{2}}{m}\right)+O\left(\epsilon^{2}\right)
$$

which implies

$$
R_{0,1} \geq R_{0,2} \Longleftrightarrow b_{1}-b_{2} \geq R_{0}\left(\nu_{1}-\nu_{2}\right) .
$$

where $R_{0}=\beta / m$. Strain 1 has higher strain-specific $R_{0}$ if and only if its advantage in transmission is bigger than its relative disadvantage in clearance rate, weighted by the global reproduction number.

For any given value $R_{0,1}$, when we fix any value of $\nu_{1}$, we can find unique value $b_{1}$ such that strain 1 is associated to $b_{1}$ and $\nu_{1}$ and has the basic reproductive number $R_{0,1}$. The same holds true for strain 2 . This is plausible because of the formulas $R_{0, i}=\frac{\beta_{i}}{m_{i}}=\frac{\beta+\epsilon b_{i}}{m+\epsilon \nu_{i}}$.

Hence, with only values of basic reproductive number $R_{0,1}$ and $R_{0,2}$, in a coinfection model, we cannot determine the long time behavior of the dynamics. Figure S1] is actually a vertical slice in each sub figure of figure S6 when we keep $\Delta \nu$ unchanged and change the value of $\Delta b$ to vary $\left(R_{0,1}, R_{0,2}\right)$. It is consistent with figure $\overline{\mathrm{S} 6}$ when the smaller $\Delta \nu$ leads to the smaller possibility of coexistence in the same range of $\Delta b$ but the possibility of the exclusion of strain 2 stays the same.
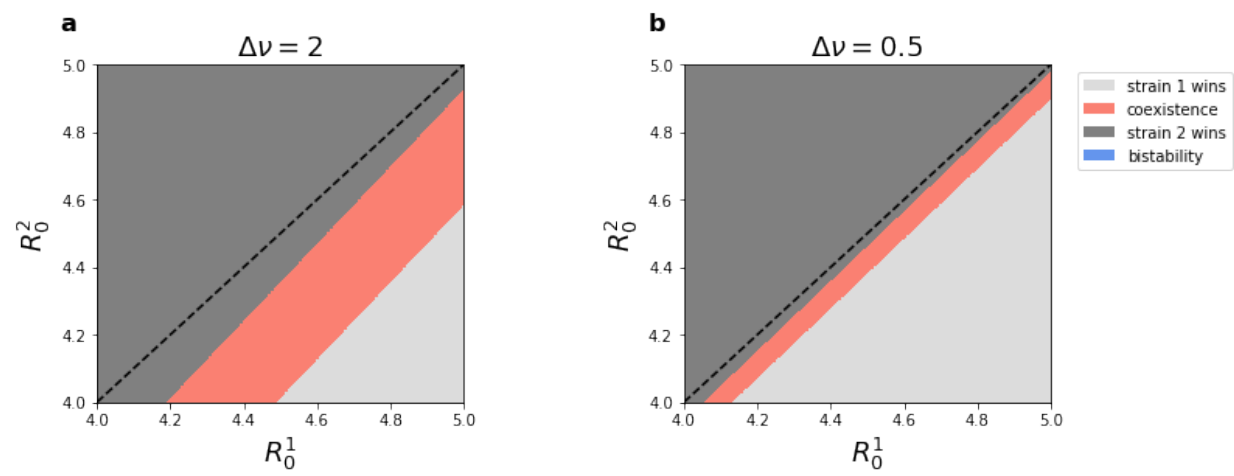

Figure S1: Ecological scenarios do not depend just on relative basic reproduction numbers of strains $R_{0,1}$ and $R_{0,2}$. We compare the case of large variation in clearance rate between two strains $\nu_{1}=-1, \nu_{2}=1$ (a) with lower one $\nu_{1}=-0.25, \nu_{2}=0.25(\mathbf{b})$. The other parameters are assumed: $k=3, m=1.5, \beta=6, \epsilon=0.1$. We vary $\Delta b$ in each case to obtain the relative comparison between $R_{0,1}$ and $R_{0,2}$ shown in the figures. The variations are in transmission rates $\beta_{i}$, single clearance rates $\gamma_{i}$, and for coinfection clearance rate we assume $\gamma_{i j}$ with $u_{12}=u_{21}=\min \left\{\nu_{1}, \nu_{2}\right\}$. Comparing (a) and (b), for the same combination of strain-specific $R_{0}$, the scenarios can diverge depending on the actual difference in strain-specific parameters leading to the particular reproduction number. Particularly, where the $\Delta \nu$ is higher (a), the relative benefit of strain 2 from lower clearance in mixed co-colonization is larger, leading to a larger strain-2 only region, and a larger coexistence region, over the same $R_{0}$ range.

\section{S2 Speed of strain dynamics depends on global parameters}

Our model allows explicit quantification of the speed of strain dynamics as a function of epidemiological parameters. Next we illustrate a dynamics example for a 2-strain system tending to exclusion. For the same relative variation between two strains, the dynamics are much faster when $R_{0}$ is lower, in this case obtained by changes in $\beta$. For the dynamics in figure $\mathrm{S2}$, we calculate the values of theta's and pairwise invasion fitnesses in each sub figure as follows: 

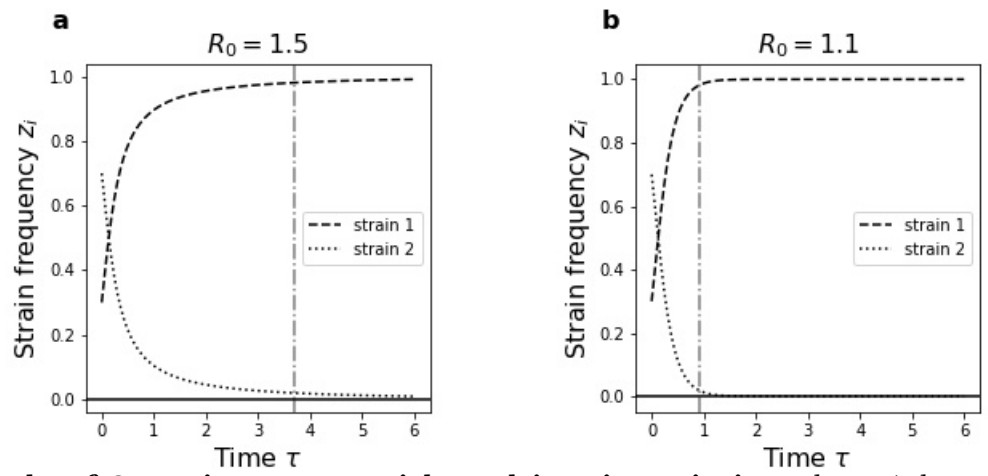

Figure S2: An example of 2-strain system with multi-trait variation. $b_{1}=1, b_{2}=-1.5, \nu_{1}=-2, \nu_{2}=-3$ $\left(u_{i j}\right)_{i j}=\left(\begin{array}{cc}1 & -2 \\ -2 & 3\end{array}\right),\left(\omega_{i j}^{i}\right)_{i j}=\left(\begin{array}{cc}-1 & -3 \\ -1 & 3\end{array}\right)$ and $\left(\alpha_{i j}\right)_{i j}=\left(\begin{array}{cc}-\sqrt{2} & 0 \\ \sqrt{2} & 0\end{array}\right)$. We choose mean transmission rate $\beta=4$, mortality $r=0.2$, mean co-colonization interaction factor (altered susceptibility to co-colonization) $k=1.5$, and the initial values of strain frequencies $\left(z_{1}, z_{2}\right)=\left(\begin{array}{ll}0.3 & 0.7\end{array}\right)$. Both figures present periods of transient coexistence before the exclusion of strain 1 . The dashed-dotted grey vertical lines, denoted by $d$ in two figures show the region where $\min \left(z_{1}, z_{2}\right)>0.02$. Figure (a) plots for $R_{0}=1.5$, in which, $d$ goes through a point between 3 and 4 (near 4 ). Figure (b) plots for $R_{0}=1.1$, in which, $d$ goes through a point very near and less than 1 . Thus, it can be seen that the the region of "effective transient coexistence" when $R_{0}=1.5$ is larger than when $R_{0}=1.1$.

- (a): $\left(\begin{array}{lllll}\theta_{1} & \theta_{2} & \theta_{3} & \theta_{4} & \theta_{5}\end{array}\right) \approx\left(\begin{array}{lllll}0.48 & 0.2 & 0.09 & 0.03 & 0.2\end{array}\right)$, and $\left(\lambda_{1}^{2}, \lambda_{2}^{1}\right) \approx(10.37,-0.20)$.

- (b): $\left(\begin{array}{lllll}\theta_{1} & \theta_{2} & \theta_{3} & \theta_{4} & \theta_{5}\end{array}\right) \approx\left(\begin{array}{lllll}0.51 & 0.39 & 0.03 & 0.07 & 0.0\end{array}\right)$, and $\left(\lambda_{1}^{2}, \lambda_{2}^{1}\right) \approx(8.28,-4.42)$.

It is easy to verify that because of the values of $\lambda_{i}^{j}$, we have exclusion of strain 2 from the system and only strain 1 persists, but depending on the value of $R_{0}$ the dynamics will be quicker or slower. In this particular case, reducing $R_{0}$, hence overall endemic prevalence of the two strains, leads to faster exclusion dynamics and shorter period of transient coexistence.

\section{S3 Qualitative transitions in the same system when varying $\mu$}

\section{S3.1 A result for variation occurring only in two out of: i) transmission rates $\beta_{i}$, ii) clearance rates $\gamma_{i}$ and ii) transmission biases from coinfection $p_{i j}^{i}$}

In this subsection, we prove that, in the case of variation between 2 strains only, can be in transmission rates $\beta_{i}$, clearance rates $\gamma_{i}$ and transmission probability from co-colonized hosts, there is at most one shift for the ecological outcome as a function of $\mu$ (single to coinfection ratio in the system). Indeed, we show this result in three cases.

\section{Case 1: Transmission rates $\beta_{i}$ and clearance rates $\gamma_{i}$ vary.}

By formulae 4.1 with $\Delta \omega=0$ we have that

$$
\left\{\begin{array}{l}
\lambda_{1}^{2}=\frac{\gamma}{2(\gamma+r)} \mu(2 \mu+1) \Delta \nu+(\mu+1)^{2} \Delta b \\
\lambda_{2}^{1}=-\lambda_{1}^{2}
\end{array} .\right.
$$

It suffices to show that the following equation can not have two positive roots

$$
\frac{\gamma}{2(\gamma+r)} \mu(2 \mu+1) \Delta \nu+(\mu+1)^{2} \Delta b=0
$$

Assume the contradiction, which means that it has two positive roots, which can be denoted by $\mu_{1}$ and $\mu_{2}$. By Viete's theorem, we have that

$$
\mu_{1}+\mu_{2}=-\frac{\frac{\gamma}{2(\gamma+r)} \Delta \nu+2 \Delta b}{\frac{\gamma}{\gamma+r} \Delta \nu+\Delta b}>0, \quad \mu_{1} \mu_{2}=\frac{\Delta b}{\frac{\gamma}{\gamma+r} \Delta \nu+\Delta b}>0
$$

Thus, S3.4 implies $\mu_{1}+\mu_{2}+\frac{3}{2} \mu_{1} \mu_{2}>0$, which is equivalent to

$$
-\frac{\frac{\gamma}{2(\gamma+r)} \Delta \nu+2 \Delta b}{\frac{\gamma}{\gamma+r} \Delta \nu+\Delta b}+\frac{\frac{3}{2} \Delta b}{\frac{\gamma}{\gamma+r} \Delta \nu+\Delta b}>0,
$$


which is absurd because the left-hand side is equal to $-\frac{1}{2}$.

Case 2: Clearance rates $\gamma_{i}$ and transmission probability from coinfected hosts $p_{i j}^{i}$ vary.

By formulae 4.1 with $\Delta b=0$ we have that

$$
\left\{\begin{array}{l}
\lambda_{1}^{2}=\frac{\gamma}{2(\gamma+r)} \mu(2 \mu+1) \Delta \nu+(\mu+1) \Delta \omega \\
\lambda_{2}^{1}=-\lambda_{1}^{2}
\end{array} .\right.
$$

It suffices to show that the following equation can not have two positive roots

$$
\frac{\gamma}{2(\gamma+r)} \mu(2 \mu+1) \Delta \nu+(\mu+1) \Delta \omega=0 .
$$

Assume the contradiction, which means that it has two positive roots, which can be denoted by $\mu_{1}$ and $\mu_{2}$. By Viete's theorem, we have that

$$
\mu_{1}+\mu_{2}=-\frac{\frac{\gamma}{2(\gamma+r)} \Delta \nu+\Delta \omega}{\frac{\gamma}{\gamma+r} \Delta \nu}>0, \quad \mu_{1} \mu_{2}=\frac{\Delta \omega}{\frac{\gamma}{\gamma+r} \Delta \nu}>0 .
$$

Thus, (S3.4) implies $\mu_{1}+\mu_{2}+\mu_{1} \mu_{2}>0$, which is equivalent to

$$
-\frac{\frac{\gamma}{2(\gamma+r)} \Delta \nu+\Delta \omega}{\frac{\gamma}{\gamma+r} \Delta \nu}+\frac{\Delta \omega}{\frac{\gamma}{\gamma+r} \Delta \nu}>0
$$

which is absurd because the left-hand side is equal to $-\frac{1}{2}$.

Case 3: Transmission probabilities from coinfected hosts $p_{i j}^{i}$ and transmission rates $\beta_{i}$ vary.

By formulae 4.1] with $\Delta \nu=0$ we have that

$$
\left\{\begin{array}{l}
\lambda_{1}^{2}=(\mu+1)^{2} \Delta b+(\mu+1) \Delta \omega \\
\lambda_{2}^{1}=-\lambda_{1}^{2}
\end{array} .\right.
$$

The quadratic equation

$$
(\mu+1)^{2} \Delta b+(\mu+1) \Delta \omega=0
$$

has one root to be -1 . Hence, it can not have two positive roots, which implies our requirement.

\section{S3.2 The case of four ecological outcomes in the same system according to $\mu$}

It is possible to have four distinct ecological outcomes between which the same system with two strains can shift, as a function of global $\mu$. Initially, we will prove that the necessary condition for the presenting of fully four survival outcomes: E1, E2, C, B is that, variations are in coinfection clearance rates $\gamma_{i j}$ and co-colonization interaction $k_{i j}$.

Proof. • Firstly, we prove that if variations are only in $\beta_{i}, \gamma_{i}, p_{i j}^{i}$ and $k_{i j}$ then we can not have four survival scenarios as $\mu \rightarrow \infty$.

The formulas for pairwise invasion fitnesses are

$$
\begin{gathered}
\lambda_{1}^{2}=\frac{\gamma}{2(\gamma+r)} \mu(2 \mu+1) \Delta \nu+(\mu+1) \Delta \omega+\frac{\left(R_{0}-1\right) \mu}{2}\left(\mu\left(\alpha_{21}-\alpha_{12}\right)+\alpha_{21}-\alpha_{22}\right)+(\mu+1)^{2} \Delta b \\
\lambda_{2}^{1}=-\frac{\gamma}{2(\gamma+r)} \mu(2 \mu+1) \Delta \nu-(\mu+1) \Delta \omega+\frac{\left(R_{0}-1\right) \mu}{2}\left(-\mu\left(\alpha_{21}-\alpha_{12}\right)+\alpha_{12}-\alpha_{11}\right)-(\mu+1)^{2} \Delta b
\end{gathered}
$$

We have that two equations $\lambda_{1}^{2}=0$ and $\lambda_{2}^{1}=0$ are respectively equivalent to

$$
\text { (Eq1): } \quad A_{1} \mu^{2}+B_{1} \mu^{2}+C_{1}=0, \quad A_{1}>0
$$

and

$$
\text { (Eq2): } \quad A_{2} \mu^{2}+B_{2} \mu^{2}+C_{2}=0, \quad A_{2}>0
$$


in which $A_{1}=A_{2}$ and $C_{1}=C_{2}$, easily deduced from (S3.6).

On the other side, by direct verification, to have fully four outcomes, the signs of $\left(\lambda_{1}^{2}, \lambda_{2}^{1}\right)$ must change at least three times, so one in two equations (Eq 1) or (Eq 2) must have two distinguished positive solutions and the other must have at least one positive solution. By Viete's theorem, the products of solutions in two equations (Eq 1) and (Eq 2) are equal. Hence, (Eq 1) and (Eq 2) must have both positive solutions. Denote two positive solutions of (Eq 1) are $x_{1}<x_{2}$, two positive solutions of (Eq 2) are $y_{1}<y_{2}$.

By direct checking, and note that when $\mu \rightarrow+\infty$ or $\mu \rightarrow-\infty, \lambda_{1}^{2}+\lambda_{2}^{1} \rightarrow 0$, we have that, to have fully four outcomes, it must be

$$
x_{1}<y_{1}<x_{2}<y_{2}
$$

or

$$
y_{1}<x_{1}<y_{2}<x_{2} .
$$

Both of these inequality requires the products of solutions in (Eq $\mathbf{1})$ is strictly less or more than the products of solutions in (Eq 2), which is a contradiction of the equality of two products mentioned before.

Thus, if variations are only in $\beta_{i}, \gamma_{i}, p_{i j}^{i}$ and $k_{i j}$ then we can not have four survival scenarios as $\mu \rightarrow \infty$.

- Secondly, we prove that if variations are only in $\beta_{i}, \gamma_{i}, \gamma_{i j}$ and $p_{i j}^{i}$ then we can not have four survival scenarios as $\mu \rightarrow \infty$.

The formulas for pairwise invasion fitnesses now read

$$
\begin{gathered}
\lambda_{1}^{2}=\frac{\gamma}{2(\gamma+r)} \mu(2 \mu+1) \Delta \nu+\frac{\gamma}{2(\gamma+r)}(\mu+1) \Delta_{2} u+(\mu+1) \Delta \omega+(\mu+1)^{2} \Delta b \\
\lambda_{2}^{1}=-\frac{\gamma}{2(\gamma+r)} \mu(2 \mu+1) \Delta \nu+\frac{\gamma}{2(\gamma+r)}(\mu+1) \Delta_{1} u-(\mu+1) \Delta \omega-(\mu+1)^{2} \Delta b
\end{gathered}
$$

For the sake of simplicity, we denote that $m:=\frac{2(\gamma+r)}{\gamma}, d_{1}:=-\nu_{12}-\nu_{21}+2 \nu_{22}$ and $-d_{2}:=-\nu_{12}-\nu_{21}+2 \nu_{11}$. We have that two equations $\lambda_{1}^{2}=0$ and $\lambda_{2}^{1}=0$ are respectively equivalent to

$$
\text { (Eq3): } \quad A_{1}^{\prime} \mu^{2}+B_{1}^{\prime} \mu^{2}+C_{1}^{\prime}=0, \quad A_{1}^{\prime}>0
$$

and

$$
(\mathbf{E q} 4): \quad A_{2}^{\prime} \mu^{2}+B_{2}^{\prime} \mu^{2}+C_{2}^{\prime}=0, \quad A_{2}^{\prime}>0
$$

now in which

$$
A_{1}^{\prime}=2 \Delta \nu+m \Delta b, \quad B_{1}^{\prime}=\Delta \nu+d_{1}+2 m \Delta b, \quad C_{1}^{\prime}=d_{1}+m \Delta b
$$

and

$$
A_{2}^{\prime}=2 \Delta \nu+m \Delta b, \quad B_{2}^{\prime}=\Delta \nu+d_{2}+2 m \Delta b, \quad C_{2}^{\prime}=d_{2}+m \Delta b .
$$

Denote two solutions of (Eq 3) to be $x_{3}<x_{4}$, and two solutions of (Eq 4) to be $y_{3}<y_{4}$. By the same arguments, it can be deduce that, to have fully four survival outcome, it must be

$$
\text { (*) } \quad x_{3}<y_{3}<x_{4}<y_{4}
$$

or

$$
(* *) \quad y_{3}<x_{3}<y_{4}<x_{4}
$$

where at least $0<x_{3}<x_{4}$ or $0<y_{3}<y_{4}$. Without loss of generality, similarly to the previous arguments, we assume that $0<x_{3}<x_{4}$ and $y_{4}>0$.

According to Viete's theorem, we obtain, note that $A_{1}^{\prime}=A_{2}^{\prime}$

$$
\begin{aligned}
x_{3}+x_{4}=-\frac{B_{1}^{\prime}}{A_{1}^{\prime}}, & x_{3} x_{4}=\frac{C_{1}^{\prime}}{A_{1}^{\prime}}, \\
y_{3}+y_{4}=-\frac{B_{2}^{\prime}}{A_{2}^{\prime}}, & y_{3} y_{4}=\frac{C_{2}^{\prime}}{A_{2}^{\prime}} .
\end{aligned}
$$

Case 1: $A_{1}^{\prime}=A_{2}^{\prime}>0$.

- If $d_{1}>d_{2}$ then $-\frac{B_{1}^{\prime}}{A_{1}^{\prime}}<-\frac{B_{2}^{\prime}}{A_{2}^{\prime}}$ and $\frac{C_{1}^{\prime}}{A_{1}^{\prime}}>\frac{C_{2}^{\prime}}{A_{2}^{\prime}}$, which implies $x_{3}+x_{4}<y_{3}+y_{4}$ and $x_{3} x_{4}>y_{3} y_{4}$. From $x_{3}+x_{4}<y_{3}+y_{4}$, then $0<x_{3}<y_{3}<x_{4}<y_{4}$, which contradicts $x_{3} x_{4}>y_{3} y_{4}$. 
- If $d_{1}<d_{2}$ then $-\frac{B_{1}^{\prime}}{A_{1}^{\prime}}>-\frac{B_{2}^{\prime}}{A_{2}^{\prime}}$ and $\frac{C_{1}^{\prime}}{A_{1}^{\prime}}<\frac{C_{2}^{\prime}}{A_{2}^{\prime}}$, which implies $x_{3}+x_{4}>y_{3}+y_{4}$ and $x_{3} x_{4}<y_{3} y_{4}$. From $x_{3} x_{4}<y_{3} y_{4}$, then $0<x_{3}<y_{3}<x_{4}<y_{4}$, which contradicts $x_{3}+x_{4}>y_{3}+y_{4}$.

Case 2: $A_{1}^{\prime}=A_{2}^{\prime}<0$.

- If $d_{1}>d_{2}$ then $-\frac{B_{1}^{\prime}}{A_{1}^{\prime}}>-\frac{B_{2}^{\prime}}{A_{2}^{\prime}}$ and $\frac{C_{1}^{\prime}}{A_{1}^{\prime}}<\frac{C_{2}^{\prime}}{A_{2}^{\prime}}$, which implies $x_{3}+x_{4}>y_{3}+y_{4}$ and $x_{3} x_{4}<y_{3} y_{4}$. From $x_{3} x_{4}<y_{3} y_{4}$, then $0<x_{3}<y_{3}<x_{4}<y_{4}$, which contradicts $x_{3}+x_{4}>y_{3}+y_{4}$.

- If $d_{1}<d_{2}$ then $-\frac{B_{1}^{\prime}}{A_{1}^{\prime}}<-\frac{B_{2}^{\prime}}{A_{2}^{\prime}}$ and $\frac{C_{1}^{\prime}}{A_{1}^{\prime}}>\frac{C_{2}^{\prime}}{A_{2}^{\prime}}$, which implies $x_{3}+x_{4}<y_{3}+y_{4}$ and $x_{3} x_{4}>y_{3} y_{4}$. From $x_{3}+x_{4}<y_{3}+y_{4}$, then $0<x_{3}<y_{3}<x_{4}<y_{4}$, which contradicts $x_{3} x_{4}>y_{3} y_{4}$.

Hence, if variations are only in $\beta_{i}, \gamma_{i}, \gamma_{i j}$ and $p_{i j}^{i}$ then we can not have four survival scenarios as $\mu \rightarrow \infty$.

In the main text, we give an example, in which varying $\mu$ from 0 to $\infty$ may give us 4 outcome exclusion of either strain, coexistence and bistability.

\section{S4 Examples for 3 possible global outcomes}

We illustrate possible outcomes as a function of $\Delta b$ and $\Delta \nu$, similarly to Figure 6, when the perturbations are only in transmission rates $\beta_{i}$, clearance rates $\gamma_{i}$ and co-colonization interactions $k_{i j}$. We recall that the borders separating exclusion regions are lines representing $\lambda_{1}^{2}=0$ and $\lambda_{2}^{1}=0$.

According to the explicit formulas of $\left(\lambda_{1}^{2}, \lambda_{2}^{1}\right)$, the border lines have the same slope, thus leading to parallelism. Thus, there are at most 3 possible outcomes for each fixed value $\left(R_{0}, k\right)$. Figures $\mathrm{S} 3$ and $\mathrm{S} 4$ shows that changing the matrix $\left(\alpha_{i j}\right)$ may generate different final outcomes.

According to 3.1), we can deduce that regardless of changing $\left(\alpha_{i j}\right)$, we can not observe coexistence and bistability for a fixed value of $\left(\alpha_{i j}\right)$. Indeed, according (3.1), two coefficients of $\Delta \nu$ in the formulae of $\lambda_{1}^{2}$ and $\lambda_{2}^{1}$ are of opposite sign, and the same holds for $\Delta b$.

Then, for $(\Delta \nu, \Delta b) \rightarrow(\infty, \infty)$ and $(-\infty,-\infty)$, we have the opposing signs of $\left(\lambda_{1}^{2}, \lambda_{2}^{1}\right)$, leading to exclusion.

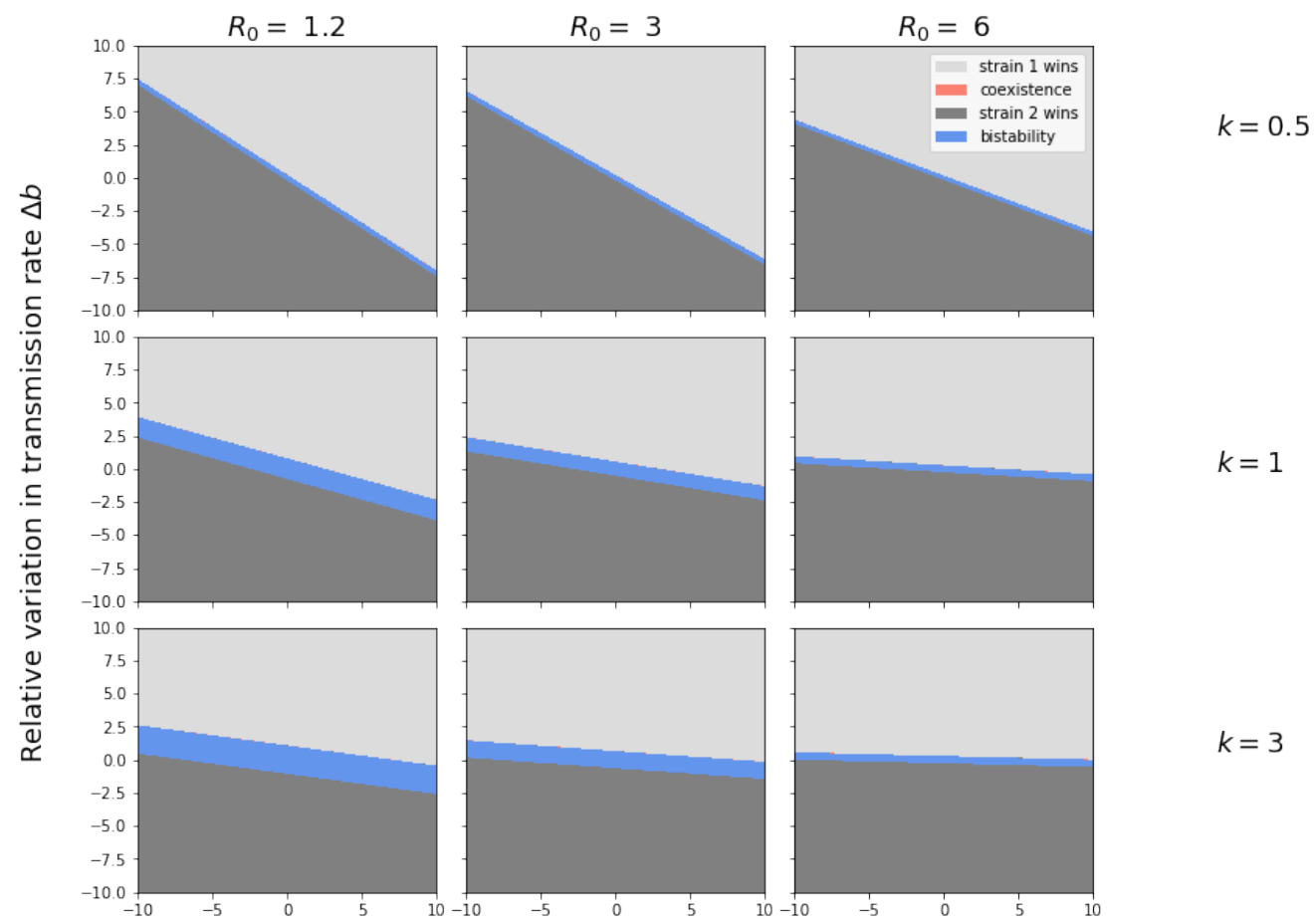

Relative variation in clearance rate $\Delta v$

Figure S3: Illustration of 3 possible outcomes, as a function of $\Delta \nu$ and $\Delta b$, for different values of $k$ and $R_{0}$. We highlight the respective regions in different colors, according to the critical relationship between $\Delta b, \Delta \nu, k$ and $R_{0}$ when perturbations happen only $\beta_{i}, \gamma_{i}$, and $k_{i j}$ with $\gamma=1, r=0.2$ and the matrix $\left(\alpha_{i j}\right)$ be $\left(\begin{array}{cc}0 & -\sqrt{2} \\ -\sqrt{2} & 0\end{array}\right)$. Three possible survival outcomes include the exclusion of strain 1, bistability and the exclusion of strain 2 . 


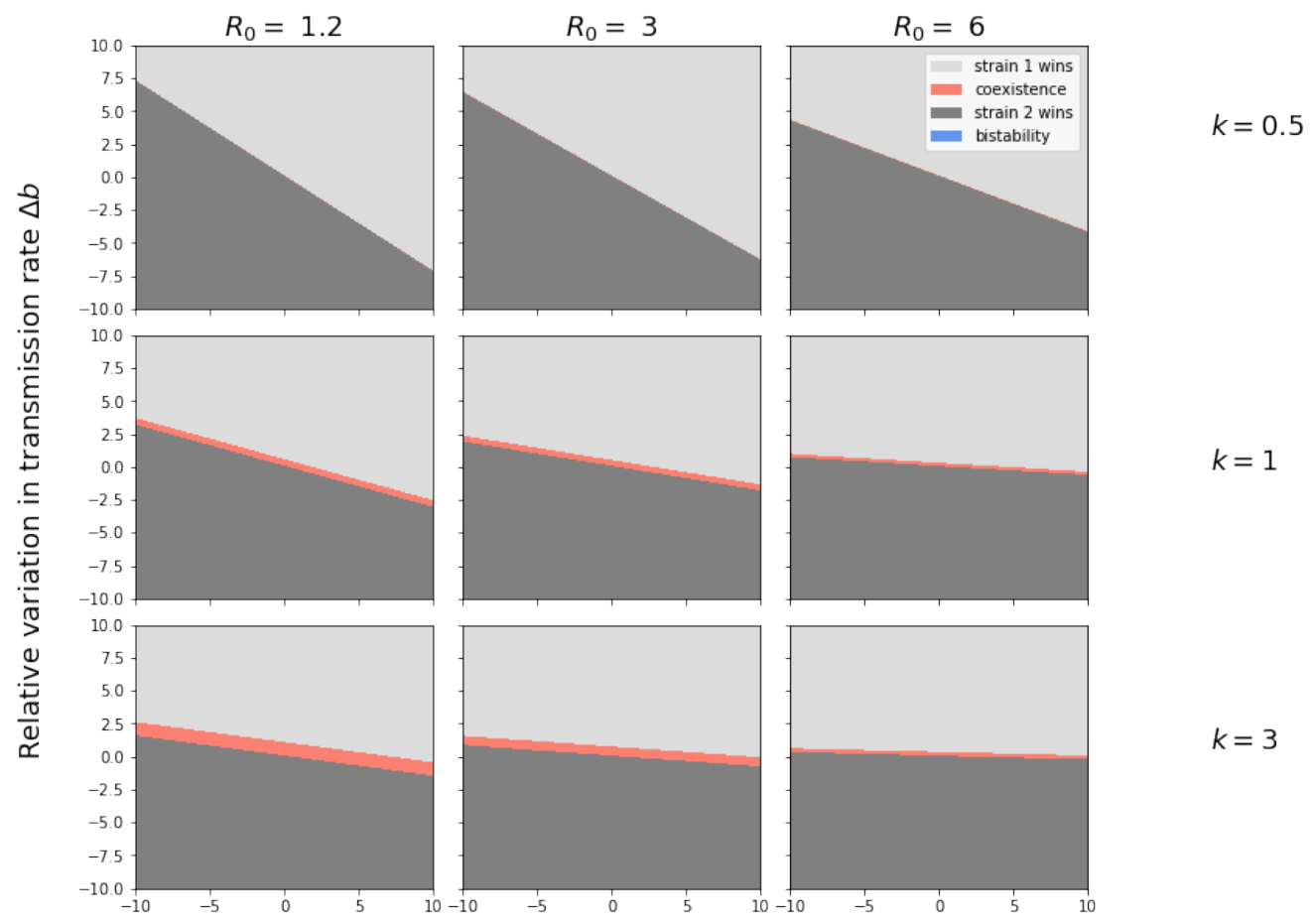

Relative variation in clearance rate $\Delta v$

Figure S4: Illustration of 3 possible outcomes, as a function of difference in transmission and clearance rates between two strains, for different values of $k$ and $R_{0}$. We highlight the respective regions in different colors, according to the critical relationship between $\Delta b, \Delta \nu, k$ and $R_{0}$ when perturbations happen only $\beta_{i}, \gamma_{i}$, and $k_{i j}$ with $\gamma=1, r=0.2$ and the matrix $\left(\alpha_{i j}\right)$ be $\left(\begin{array}{cc}-2 & 0 \\ 0 & 0\end{array}\right)$. This changing of matrix $\left(\alpha_{i j}\right)$ flips the position of two lines $\lambda_{1}^{2}=0$ and $\lambda_{2}^{1}=0$ leads to the other possible outcomes. Three possible system outcomes include: the exclusion of strain 1 , coexistence and the exclusion of strain 2 .

\section{S5 The analytical expression for coexistence prevalence}

Here we compute the value of $z_{2}^{*}$ in the case of symmetric co-colonization interaction matrix. Thus: $\alpha_{i j}=\alpha_{j i}$, $\alpha_{i i}=\alpha_{j j}$. We also assume that strain-specific transmission probability from mixed coinfected hosts is in this case $p_{i j}^{i}=\frac{1}{2}$ (no priority effects).

- Perturbation in $\beta_{i}, \gamma_{i}, \gamma_{i j}, k_{i j}$

$$
z_{2}^{*}=\frac{1}{2} \cdot \frac{-\Delta b-\frac{\theta_{2}}{\theta_{1}} \Delta \nu+\frac{\theta_{3}}{\theta_{1}}\left(2 u_{11}-u_{12}-u_{21}\right)+\frac{\theta_{5}}{\theta_{1}}\left(\alpha_{12}-\alpha_{11}\right)}{\frac{\theta_{3}}{\theta_{1}}\left(u_{11}+u_{22}-u_{12}-u_{21}\right)+\frac{\theta_{5}}{\theta_{1}}\left(\alpha_{12}-\alpha_{11}\right)}
$$

- Perturbation in $\beta_{i}, \gamma_{i}, k_{i j}$

$$
\begin{aligned}
z_{2}^{*} & =\frac{1}{2} \cdot \frac{-\Delta b-\frac{\theta_{2}}{\theta_{1}} \Delta \nu+\frac{\theta_{5}}{\theta_{1}}\left(\alpha_{12}-\alpha_{11}\right)}{\frac{\theta_{5}}{\theta_{1}}\left(\alpha_{12}-\alpha_{11}\right)}=\frac{1}{2}\left(\frac{-\Delta b-\frac{\theta_{2}}{\theta_{1}} \Delta \nu}{\frac{\theta_{5}}{\theta_{1}}\left(\alpha_{12}-\alpha_{11}\right)}+1\right) \\
& =\frac{1}{2}\left(-\frac{1}{\alpha_{12}-\alpha_{11}} \cdot \frac{2(\gamma+r)+k(\beta-\gamma-r)}{k(\beta-\gamma-r)^{2}} \cdot \gamma \Delta \nu-\frac{1}{\alpha_{12}-\alpha_{11}} \cdot \frac{2(\gamma+r+k(\beta-\gamma-r))^{2}}{k(\beta-\gamma-r)^{2}} \Delta b+1\right) .
\end{aligned}
$$




\section{S5.1 Studying the monotonicity of $z_{2}^{*}$ : increasing vs. decreasing equilibrium strain frequency}

For the sake of simplicity, and for the purposes of illustration, we consider the equilibrium of resistance strain in section 4 . Recall the formula of $z_{2}^{*}$ in $(5.8)$

$$
z_{2}^{*}=\frac{1}{2}-\Delta \nu \cdot \gamma \frac{2(\gamma+r)+k(\beta-\gamma-r)}{2 \sqrt{2} k(\beta-\gamma-r)^{2}}-\Delta b \frac{[\gamma+r+k(\beta-\gamma-r)]^{2}}{\sqrt{2} k(\beta-\gamma-r)^{2}} .
$$

To investigate the monotonicity of strain frequency, we need to compute the first partial derivative of $z_{2}^{*}$ with respect to $\beta$ and $\gamma$, noting that $m=\gamma+r$ and investigate its sign:

$$
\frac{\partial z_{2}^{*}}{\partial \beta}=-\Delta \nu \frac{1}{2 \sqrt{2} k} \frac{(k-4) m-\beta k}{(\beta-m)^{3}}-\Delta b \frac{1}{\sqrt{2} k} \frac{2 m((k-1) m-\beta k)}{(\beta-m)^{3}},
$$

and

$$
\frac{\partial z_{2}^{*}}{\partial \gamma}=-\Delta \nu \frac{1}{2 \sqrt{2} k} \frac{\beta(\beta k-k m+4 m)-r((k+2) \beta-(k-2) m)}{(\beta-m)^{3}}-\Delta b \frac{1}{\sqrt{2} k} \frac{2 \beta(\beta k-k m+m)}{(\beta-m)^{3}} .
$$

It can be seen that the expressions are entirely explicit but they display nonlinear dependence on many parameters, including strain variation $(\Delta b, \Delta \nu)$, as well as mean parameters $\beta, m$ or coinfection determinants ( $k$, etc.). This allows to obtain a full analytic understanding of the coexistence between two strains at the epidemiological level and how their relative hierarchies depend and may shift with overall context.
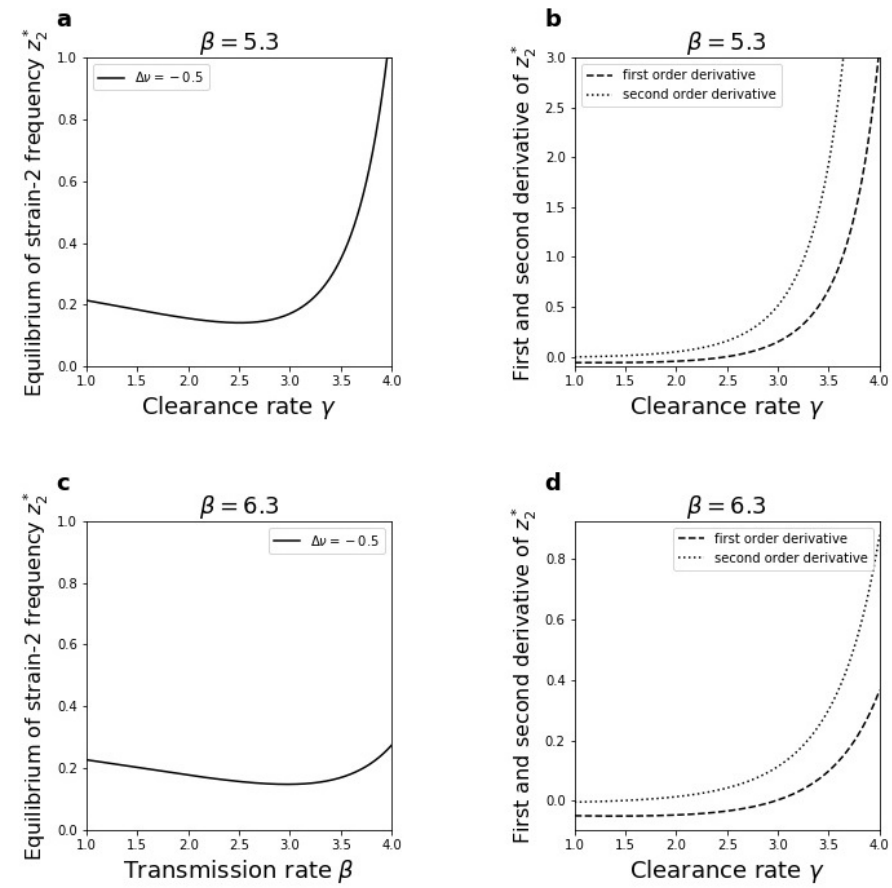

Figure S5: Monotonicity and convexity of $z_{2}^{*}$ according to transmission rate $\beta$ and clearance rate $\gamma$. We choose $r=0.2, k=1$ and matrix including entries $\alpha_{i j}$ is $\left(\begin{array}{cc}0 & \sqrt{2} \\ \sqrt{2} & 0\end{array}\right)$. The behavior observed is by plotting for $\Delta \nu=-0.5$. In (a) and (c), setting $\Delta b=0.3$, we plot $z_{2}^{*}$ according to $\gamma$ with $\beta=5.3$ and $\beta=6.3$ respectively. Figures (c) and (b) present the curve of first and second orders derivatives of $z_{2}^{*}$ according to $\gamma$, respective for figures (a) and (b).

\section{S5.2 Studying the convexity of $z_{2}^{*}$ : accelerating vs. decelerating behavior}

In this subsection, we consider the convexity of $z_{2}^{*}$ with respect to $\beta$ and $\gamma$, global strain-transcending parameters, that can be controlled via interventions (antibiotic treatment, vaccines, etc.). Thus, we compute the second-order derivative of $z_{2}^{*}$ related to $\beta$ as follows, noting that $m=\gamma+r$ :

$$
\frac{\partial^{2} z_{2}^{*}}{\partial \beta^{2}}=-\Delta \nu \frac{1}{\sqrt{2} k} \frac{6 \beta m+(-4 m+k)(\beta-m)}{(\beta-m)^{4}}-\Delta b \frac{\sqrt{2} m(3 m-2 k m+2 \beta k)}{k(\beta-m)^{4}},
$$


and the second-order derivative of $z_{2}^{*}$ related to $\gamma$ as follows:

$$
\frac{\partial^{2} z_{2}^{*}}{\partial \gamma^{2}}=-\Delta \nu \frac{\beta k-(k-6) m}{\sqrt{2} k(\beta-m)^{4}}-\Delta b \frac{2 \beta((2 k+1)-2(k-1) m)}{k(\beta-m)^{4}} .
$$

These expressions reveal whether the behavior of the strain frequency as a function of global parameters is decelerating or accelerating. This has implications for the control of the strain composition at the population level if we intervene via changing global transmissibility of all strains $\beta$ or global clearance rate $\gamma$. If the behavior is increasing and accelerating, this means that the frequency of that strain can reach fixation for some value of the global parameter. If the behavior is increasing and decelerating (first derivative positive and second derivative negative) this means that the strain frequency saturates at a particular value $<1$ indicating coexistence in the limit of high values of the control parameter. The following simulation in Figure S5 allows us to see how we can use these analytical expressions above to predict exactly the behavior the strain coexistence frequencies as a function of our parameters, and possible responses to interventions in global transmission rate $\beta$ or clearance rate $\gamma$. In these figures, we can see that $z_{2}^{*}$ is always convex related to $\gamma$ in the considered period of varying $\gamma$.

\section{S5.3 Effect of transmission priority effects from mixed coinfection $\Delta \omega$}

To see the effect of transmission biases from mixed coinfection, as in the section 4 we plot the values of pairwise invasion fitnesses according to the single to co-colonization ratio $\mu$. From 4.1, it is known that if variations are only in transmission rates $\beta_{i}$, clearance rates $\gamma_{i}$ and transmission probability from coinfected hosts $p_{i j}^{i}$, the outcome is always the exclusion of either strain. In this figure, the perturbations are in transmission rates, clearance rates, transmission probability from co-colonized hosts as well as in co-colonization interaction factor $k_{i j}$ to break the anti-symmetry. Except the transmission biases from mixed coinfection $p_{i j}^{i}$ favor to strain 2 , other trait differences in $\beta_{i}, \gamma_{i}$ favors to strain 1.

We consider in three cases including co-colonization interaction factor $k_{i j}$ favors to strain 1 , disfavors to strain 2 and counter balance. Although the difference $\Delta \omega$ is bias to strain 2 , when $\mu$ is large enough, the difference in transmission probability does not effect too much. This can be seen in the similar trending of $\left(\lambda_{1}^{2}, \lambda_{2}^{1}\right)$ in figures $(\mathbf{a}, \mathbf{b}, \mathbf{c})$ in figure 4 , whose values of $\Delta b$ and $\Delta \nu$ are kept the same.

These phenomena are plausible because the weight (written in $\mu$ ) of transmission probability from coinfected hosts is $\mu+1$ in the formulae of invasion fitnesses 4.5 . Of course, unlike 4.3, when $\mu$ is small enough, $\Delta \omega$ leads to the exclusion of strain 2 in figure $\mathrm{S} 6$ (a) and the exclusion of strain 1 in figure S6 (b). Meanwhile, $\lambda_{1}^{2}$ is always positive in figure 4 (a) and $\lambda_{2}^{1}$ is always negative in figure 4 (b).
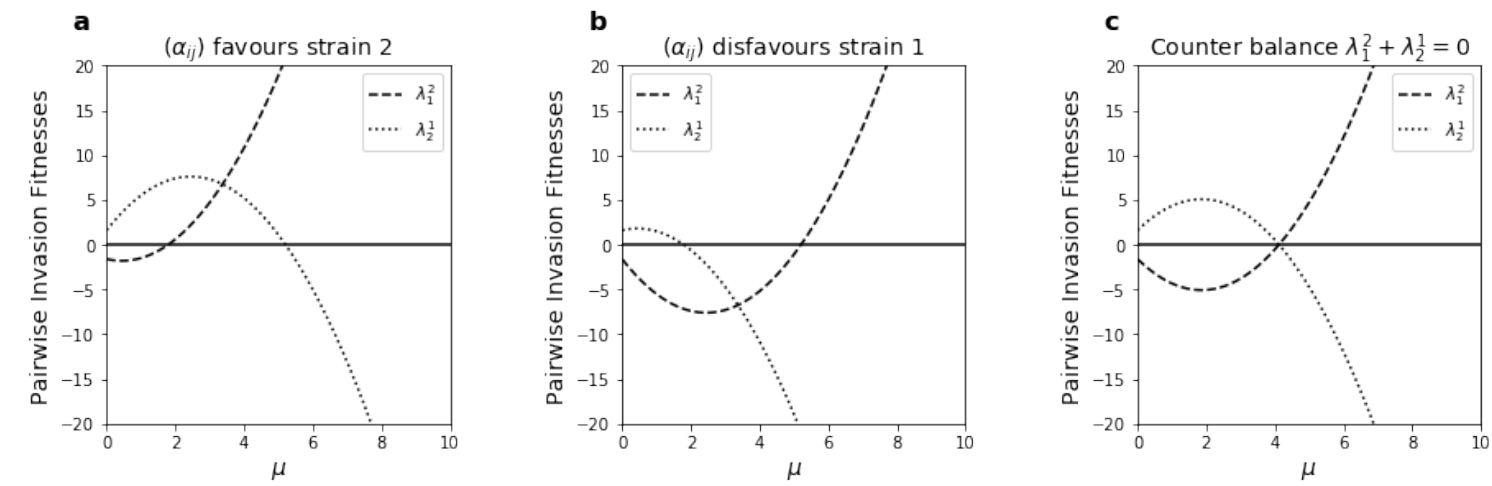

Figure S6: Additional effects of within-host transmission advantage from mixed coinfection $p_{i j}^{i} \neq \frac{1}{2}$ (for comparison with Figure 4 a-b-c) Here we combine variation in co-colonization interactions $k_{i j}$ with variations in transmission rates, infection clearance rates, and transmission probability from coinfected hosts (See Eqs (4.9)). We compute pairwise invasion fitnesses $\left(\lambda_{1}^{2}, \lambda_{2}^{1}\right)$ according to $\mu$ in various cases of co-colonization interaction matrix $\left(\alpha_{i j}\right)$. We illustrate the cases of transmission and clearance superiority of strain 1 (parameters as in top row of Figure 4): $\Delta b=0.4, \Delta \nu=0.8, R_{0}=5, r=0.5$ and $\gamma=1.5$. We choose the value $\Delta \omega=-2$ to increase the advantage in transmission probability of strain 2 from mixed coinfection. We consider 3 structures: (a) $\left(\begin{array}{cc}-2 & 0 \\ 0 & 0\end{array}\right) ;(\mathbf{b})\left(\begin{array}{cc}0 & 0 \\ 0 & 2\end{array}\right)$; (c) $\left(\begin{array}{cc}-\sqrt{2} & 0 \\ 0 & \sqrt{2}\end{array}\right)$ for variation in co-colonization interactions.

Secondly, as mentioned in Sections 6.1 and 6.2, we point out that depending on how the fitness differential between strain 1 and its competitor strain 2 , is manifested $(\Delta b, \Delta \nu)$ increasing strain-transcending clearance or tranmission rates $(\gamma$ or $\beta)$ in a population can have opposing effects. Besides other trait parameters which are kept unchanged, we analyze the effect of $\Delta \omega<0$ favouring strain 2 , and how this modifies the range 
of $\gamma$ guaranteeing the coexistence, originally observed in Figure S7. We can see that the shapes of curves representing $z_{2}^{*}$ are similar to the Figures $8(\mathbf{a}, \mathbf{b}, \mathbf{c}, \mathbf{d})$. respectively. However, in Figures $\mathbf{S 7}$ for all $(\mathbf{a}, \mathbf{b}$, $\mathbf{c}, \mathbf{d}$ ), for $\Delta \nu>0$ (which favours strain 1 ), compared to figures $8(\mathbf{a}, \mathbf{b}, \mathbf{c}, \mathbf{d}$ ) respectively, the ranges for survival of strain 2 are larger, highlighting the positive effect of its precedence in transmission from mixed coinfection. In contrast to this, when $\Delta \nu<0$, the range for coexistence decreases, to a larger advantage of strain 2-only persistence. In all cases, the values of equilibrium of strain 2 in Figure S7 corresponding to each parameter $\gamma$ or $\beta$ are higher than in Figure 8 .
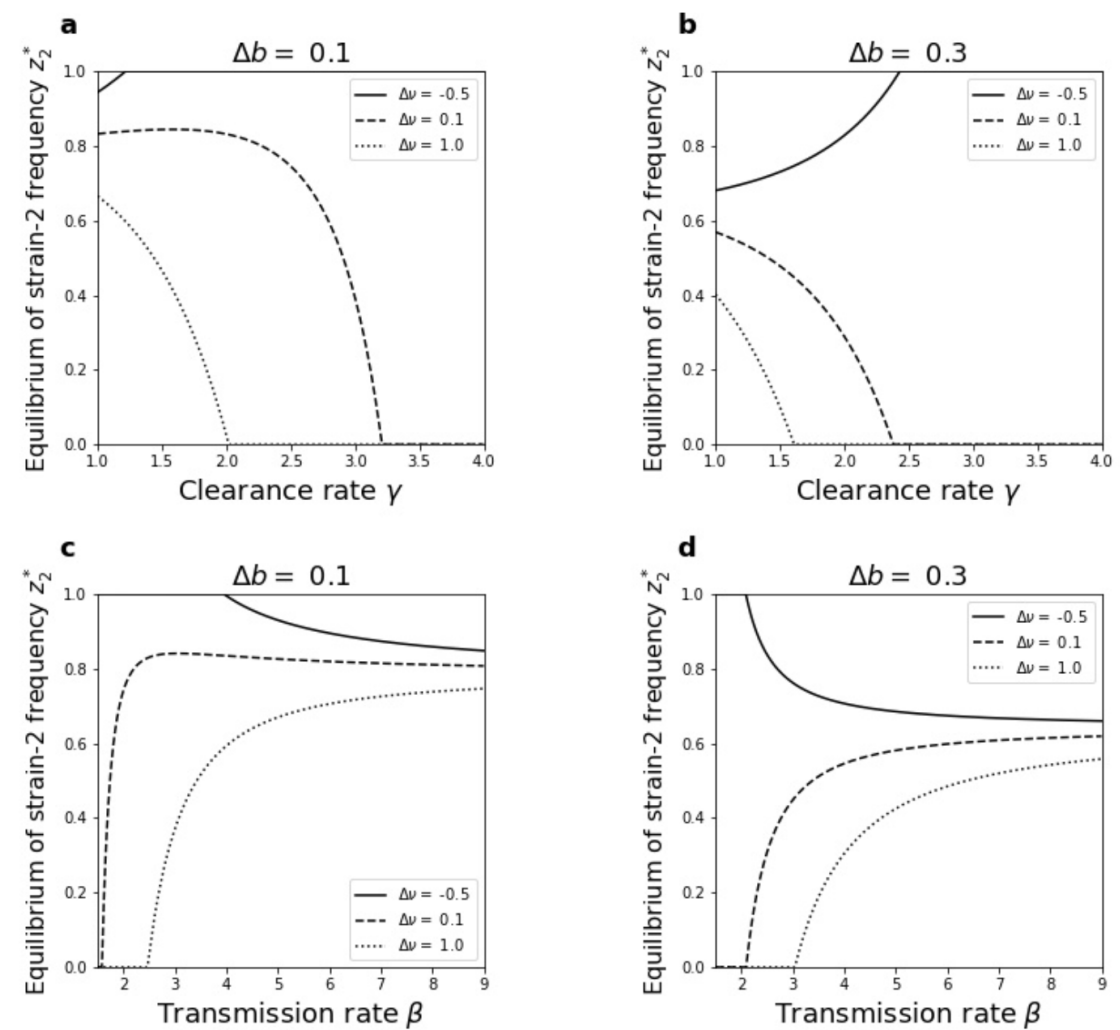

\section{Intervention}

Figure S7: Strain frequency at the endemic equilibrium vs. $\gamma$ and $\beta$ depends on underlying trait variation (related to Fig 8 but with $\Delta \omega \neq 0$ ). We plot the prevalence of strain 2, $z_{2}^{*}$ at the coexistence equilibrium (Eq. 5.8), as a function of mean transmission rate and mean clearance rate. Variation among 2-strains is encoded in the transmission and clearance rate axes: $\beta_{i}$ and $\gamma_{i}$, transmission probability from co-colonized hosts $p_{i j}^{i}$ and co-colonization vulnerabilities $k_{i j}$. In this simulation, we keep the same values of $r, k$ and the matrix of standardized interactions in Figure 8. We choose $\Delta \omega=-0.5$, which favors strain 2 in precedence of transmission from mixed coinfection. a-b. We plot the equilibrium $z_{2}^{*}$ as a function of mean clearance rate $\gamma$ (varied between 1 and 4 ) for 2 cases of fitness differentials in transmission $\Delta b$ and 3 cases of variability in clearance $\Delta \nu$. The global transmission rate is $\beta=4.5$ to ensure $R_{0} \geq 1$. c-d. We plot the equilibrium frequency of strain $2, z_{2}^{*}$, as a function of mean transmission rate $\beta$ (varied between 1.5 and 9) for 2 cases of different variation $\Delta b$ and 3 cases of $\Delta \nu$. In these plots, overall clearance rate is held fixed at $\gamma=1$ to ensure $R_{0} \geq 1$. 